\title{
A Comparative Study on Acoustic Optimization and Analysis of CLD/Plate in a Cavity Using ESO and GA
}

\author{
Dongdong Zhang $\mathbb{D}^{1}{ }^{1}$ Shuwen Wang, ${ }^{1}$ and Ling Zheng $\mathbb{D}^{2}$ \\ ${ }^{1}$ Mechanical Engineering College, University of Shanghai for Science and Technology, Shanghai, China \\ ${ }^{2}$ State Key Laboratory of Mechanical Transmission, Chongqing University, Chongqing, China \\ Correspondence should be addressed to Dongdong Zhang; dongdongzhang@usst.edu.cn
}

Received 30 June 2017; Revised 3 January 2018; Accepted 13 February 2018; Published 19 March 2018

Academic Editor: Marcello Vanali

Copyright (c) 2018 Dongdong Zhang et al. This is an open access article distributed under the Creative Commons Attribution License, which permits unrestricted use, distribution, and reproduction in any medium, provided the original work is properly cited.

\begin{abstract}
An acoustic radiation model of a cavity with a flexible plate treated with constrained layer damping (CLD) is developed by a combination of finite element method (FEM) and boundary element method (BEM). An acoustic topology optimization model is established with the objective of minimizing sound radiation power at specific modal frequency and design variables defined as locations of CLD treatments. The evolutionary structural optimization (ESO) method and genetic algorithm (GA) are employed to search optimal CLD configurations. Sound power sensitivity for CLD/plate is derived to determine search direction in ESO optimization procedure. The optimal CLD layouts for the flexible plate with two different boundary conditions are obtained and analyzed. Computational time, optimal layouts, and minimum sound power obtained using ESO and GA are compared. The results demonstrate effectiveness of the two methods, and ESO is more efficient to obtain deterministic and more practical optimal CLD material layouts for minimizing sound radiation power. The influences of CLD materials thickness and exciting force locations on optimal results obtained using ESO are discussed in detail. It is shown that the optimal rejection ratio varies with thicknesses of CLD materials and distribution of normal velocity of the flexible plate. Variation trend of the optimal rejection ratio is opposite for the two boundary conditions.
\end{abstract}

\section{Introduction}

Vibration and acoustic radiation control are important issues in many engineering applications, such as aerospace and automotive industries. Constrained layer damping (CLD) treatment has been regarded as an effective means for suppressing vibration of the flexible engineering structures and their acoustic radiation since it was presented by Kerwin [1]. However, it is not practical and economical to be full coverage CLD treatment on the base structure due to the limitation of added weight. For example, in automotive and airplane industries, the weight induced by suppression of vibration and noise is limited in a small range. Therefore, the optimal CLD configurations for suppressing vibration and noise are pursued owing to constraints of cost and weight in engineering structures.

Zheng et al. [2, 3] utilized a genetic algorithm with penalty function to obtain optimal locations and length of the CLD patches for minimizing structural displacements of beams and cylindrical shells. Chen and Huang [4] presented a study on optimization of CLD placements for suppressing vibration of plates to minimize structural damping ratio while the shift of resonant frequency and the thickness of CLD were considered as penalty functions. A topographical method in conjunction with the complex method is used to search for the global minimum. Alvelid [5] proposed a shape optimization method by adding CLD materials to the base structure piece by piece at permissible positions in a mosaic manner with the objective function as the square of the normal surface velocity. Zhang and Zheng [6] established a multiobjective optimization configuration to get optimal thickness and locations of CLD pieces on the base plate, which can supply multiple selections of CLD treatments for different vibration control objectives and constrained conditions. Many topology optimization methods were also applied to get the optimal configuration of CLD structure. 


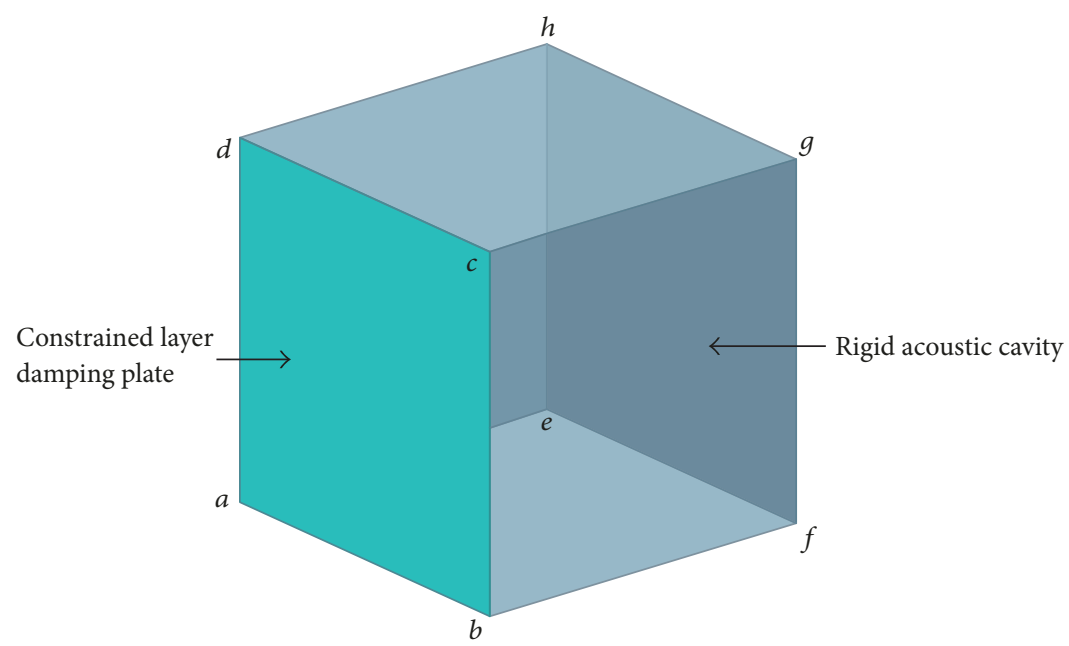

FIGURE 1: Schematic drawing of a closed acoustic cavity.

Ansari et al. [7] considered modal loss factors of a cantilever CLD/plate as objective function and proposed a novel level set method to search optimal shapes and locations of CLD patches. It shows that modal loss factor increases while the shape changes from a square to a circle. Li et al. [8] studied topology optimization problem for vibration reduction of flexible plate treated with CLD, in which evolutionary structural optimization (ESO) method is used to minimize structural modal damping ratios. Fang and Zheng [9] employed moving asymptote (MMA) method to obtain optimal layouts of CLD material on base structure subjected to harmonic excitations or stationary random excitations, in which the solid isotropic material with penalization (SIMP) method was adopted to interpolate the constrained layer damping material.

The above works on CLD optimization mainly focus on vibration suppression of flexible structures. However, the minimization of vibration response cannot warrant a minimum sound power and fewer studies have considered optimal CLD configurations for acoustic problems. Zheng and Cai [10] employed a genetic algorithm with penalty function to search the optimal CLD locations/length to minimize the sound power radiated by a simply supported baffled beam. Frequency response functions at different beam locations were used for calculation of their radiated sound power into half free-space by the use of Rayleigh's integral. Meanwhile the procedure was not suitable for complex structures as analytical models are not available. Zheng et al. [11] presented a topology optimization procedure for CLD/plate with a SIMP model. The radiated sound power, which was simplified by using sound radiation mode and modal strain energy method, was selected as objective function. Numerical and experimental results showed that the volume velocity should be also concerned besides the structural damping, for minimizing sound radiation power at low frequency range.

As that in the above literatures, it can be formulated as a topology optimization problem where the structure is designed optimally to achieve vibration or sound radiation reduction. Du and Olhoff [12] applied topology optimization to minimize the radiated sound power from bimaterial elastic continuum structures using a solid isotropic material with penalization (SIMP) interpolation model. Akl et al. [13] attempted to attenuate the structural vibration and sound pressure simultaneously inside an acoustic cavity by topology optimization of the plate coupled with the cavity. The coupling between the structure and the fluid domains is selected as the objective function. Zheng et al. [14] developed a numerical sound radiated model of a flexible CLD/plate in a rigid cavity, and genetic optimization (GA) was used to obtain optimal CLD layouts pasted on the plate to suppress sound pressure and power. The numerical results were validated experimentally.

In this paper, the work is extended based on [14]. Firstly, the formulations of acoustic radiation model of a cavity with a flexible CLD/plate are briefly resumed. Then a topology optimization problem for minimizing sound radiation power is developed, and evolutionary structural optimization (ESO) procedure is extended to solve this problem. Sound power sensitivity with respect to locations of CLD materials is formulated to lead the search direction in ESO procedure.

Numerical example is demonstrated and discussed, in which the two different boundary conditions are considered for the CLD/plate in the cavity. The emphasis is a comparison on computational time, optimal CLD layouts, and minimum sound power obtained by implementing GA optimization procedure in [14] and ESO procedure. Furthermore, the influences of CLD materials thickness and exciting force locations on optimal results are discussed in detail. Finally, a brief summary of the conclusions is given.

\section{Acoustic Radiation Model Formulation}

Acoustic radiation model of a closed cavity is formulated. Figure 1 shows a schematic drawing of the cavity. One of six surfaces of the cavity is composed of a flexible plate treated with CLD materials, while the other five are rigid. The normal velocities induced by the force applied on the CLD/plate are derived by finite element method. Then the sound power 

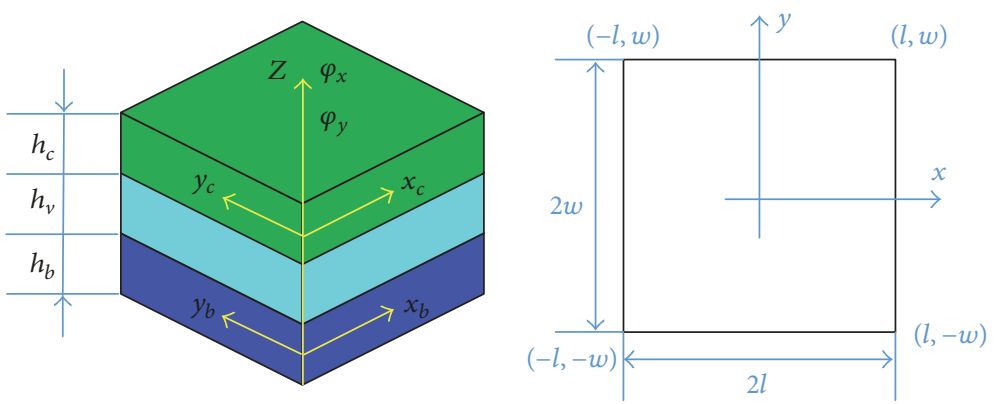

Figure 2: Composite element for CLD/plate.

radiating into the cavity is formulated by taking vibration velocities as boundary condition. The above formulations can be found in [14] in detail and briefly resumed in this section.

2.1. Formulations for Normal Velocities of CLD/Plate. Finite element method is employed to obtain the normal vibration velocities of CLD/plate. A composite element shown in Figure 2 is employed, which has four nodes with 7 degrees of freedom per node. The general displacements vector of the element can be written as

$$
\left\{\begin{array}{lllllll}
x_{c} & y_{c} & x_{b} & y_{b} & z & \varphi_{x} & \varphi_{y}
\end{array}\right\}^{T}=\mathbf{S} \mathbf{X}^{e}
$$

in which $c, b$ denote constrained layer and base plate; $x, y$ are longitudinal displacements; $z, \varphi_{x}, \varphi_{y}$ represent transverse displacement and slopes of the transverse displacements; $\mathbf{X}^{e}$ is the displacement vector of the composite element nodes. $\mathbf{S}=\left\{\begin{array}{lllllll}\mathbf{S}_{x c} & \mathbf{S}_{y c} & \mathbf{S}_{x b} & \mathbf{S}_{y b} & \mathbf{S}_{z} & \mathbf{S}_{z, x} & \mathbf{S}_{z, y}\end{array}\right\}^{T}$ is shape function matrices.

Furthermore, for the damping layer, shape functions can be derived as follows:

$$
\begin{aligned}
& \mathbf{S}_{i v}=\frac{1}{2}\left(\mathbf{S}_{i c}+\mathbf{S}_{i b}+\frac{h_{c}-h_{b}}{2} \mathbf{S}_{z, i}\right) \quad(i=x, y), \\
& \mathbf{S}_{\gamma i v}=\frac{1}{h_{v}}\left[\mathbf{S}_{u c}-\mathbf{S}_{u b}+\left(\frac{h_{c}+h_{b}}{2}+h_{v}\right) \mathbf{S}_{w, i}\right] \\
&(i=x, y)
\end{aligned}
$$

in which $v$ denotes damping layer, and $h$ denotes the thickness.

Hence, element mass matrices and stiffness matrices can be obtained based on energy method, as follows:

$$
\begin{aligned}
\mathbf{M}_{j}^{e}= & \rho_{j} h_{j} \int_{-l}^{l} \int_{-w}^{w}\left(\mathbf{S}_{x j}^{T} \mathbf{S}_{x j}+\mathbf{S}_{y j}^{T} \mathbf{S}_{y j}+\mathbf{S}_{z}^{T} \mathbf{S}_{z}\right) d x d y \\
& (j=b, c, v), \\
\mathbf{K}_{j}^{e}= & h_{j} \int_{-l}^{l} \int_{-w}^{w} \mathbf{B}_{j}^{T} \mathbf{D}_{j} \mathbf{B}_{j} d x d y \\
& +\frac{h_{j}^{3}}{12} \int_{-l}^{l} \int_{-w}^{w} \mathbf{B}_{j}^{T} \mathbf{D}_{j} \mathbf{B}_{j} d x d y \quad(j=b, c, v) .
\end{aligned}
$$

The element stiffness matrix of damping layer corresponding to shear deformation can be derived as

$$
\mathbf{K}_{\gamma v}^{e}=G h_{v} \int_{-l}^{l} \int_{-w}^{w}\left(\mathbf{S}_{\gamma x v}^{T} \mathbf{S}_{\gamma x v}+\mathbf{S}_{\gamma y v}^{T} \mathbf{S}_{\gamma y v}\right) d x d y .
$$

Finally, assembling the element matrices and exciting force, the global motion equation of CLD/plate is formulated as

$$
\mathbf{M X}+\mathbf{K X}=\mathbf{F}
$$

As a harmonic force is assumed, the vibration displacement and excitation force are reasonably expressed into $x(t)=X e^{i \omega t}, f(t)=F e^{i \omega t}$, respectively, in which $\omega$ is the excitation frequency. Equation (6) is rewritten as

$$
\left(-\omega^{2} \mathbf{M}+\mathbf{K}\right) \mathbf{X}(\omega)=\mathbf{F} .
$$

According to (6), the normal velocity vector of CLD/plate is formulated as

$$
\mathbf{V}=i \omega \mathbf{X}_{z}=i \omega \mathbf{H}(\omega) F
$$

in which $\mathbf{H}(\omega)=\left(-\omega^{2}[\mathbf{M}]+[\mathbf{K}]\right)^{-1}$ represents transfer function of the vibration displacement in frequency domain; $\mathbf{X}_{z}$ is the normal displacement vector.

2.2. Formulations for Acoustic Radiation Power of Closed Cavity. Boundary element method is employed to formulate acoustic radiation power of the closed cavity. The acoustic field composed of an ideal homogeneous air is assumed. The steady state sound pressure in the field satisfies the following equation over inner enveloping surface $S$ (boundary surface) of the cavity [15]:

$$
\begin{aligned}
C(\alpha) P(\alpha)= & \frac{1}{2 \pi} \iint_{S} \frac{\partial P(\beta)}{\partial n}\left\{\frac{e^{-i k r(\alpha, \beta)}}{r(\alpha, \beta)}\right\} d S \\
& -\frac{1}{2 \pi} \iint_{S} P(\beta) \frac{\partial}{\partial n}\left\{\frac{e^{-i k r(\alpha, \beta)}}{r(\alpha, \beta)}\right\} d S,
\end{aligned}
$$

where $P$ is the sound pressure and $k$ is the wave number; $r(\alpha, \beta)$ is the distance between two points $\alpha$ and $\beta$; $\alpha$ is 
a point inside acoustic field, and $\beta$ is a point on surface $S$; the coefficient $C(\alpha)$ is equal to 2 when $\alpha$ is inside the field, and $C(\alpha)$ is equal to 1 when $\alpha$ is on the boundary. The loading effect of the sound pressure on the structure is neglected, and no absorption effect exists on the cavity surfaces. Hence, the boundary conditions in the acoustic analysis are specified as

$$
\frac{\partial P}{\partial n}= \begin{cases}0 & \text { on the boundary surface corresponding to rigid surfaces } \\ -i \omega \rho V_{a} & \text { on the boundary surface corresponding to the CLD/plate }\end{cases}
$$

where $\rho$ and $V_{a}$ are air density and normal velocity of the air at the boundary, respectively. Based on the assumption that no absorption effect exists on the cavity surfaces, $V_{a}$ is equal to the normal vibration velocity $V_{s}$ of CLD/plate.

To obtain acoustic power radiated into the cavity, sound pressure of point $\alpha$ on boundary surface $S$ is firstly numerically calculated by the discretization of (8). Firstly, the boundary surface $S$ is divided into six subsurfaces, named $S_{1}$, $S_{2}, S_{3}, S_{4}, S_{5}$, and $S_{6} ; S_{1}$ is assumed to correspond to CLD/plate of the cavity, and the other five subsurfaces correspond to the rigid surfaces $S_{f e h g}, S_{b c g f}, S_{a e h d}, S_{a b f e}$, and $S_{d c g h}$. Hence, the discretization of (8) can be developed as follows:

$$
\begin{aligned}
P(\alpha)= & \frac{1}{2 \pi} \int_{S_{1}}\left(-i \rho \omega V_{a}(\beta)\right) \frac{e^{-i k r}}{r} d S \\
& +\frac{1}{2 \pi} \sum_{j=1}^{6} \int_{S_{j}} P(\beta) \frac{(i k r+1) e^{-i k r}}{r^{2}} \frac{\partial r}{\partial n} d S .
\end{aligned}
$$

Furthermore, every subsurface is meshed with $N$ boundary elements, shown in Figure 3. Then (9) is integrated on the boundary elements and the point $\alpha$ is supposed to be located on a node $n$ of the boundary element $m$. So (10) can be rewritten as

$$
\begin{aligned}
& P(n)=\frac{1}{2 \pi} \\
& \quad \sum_{m=1}^{N} \sum_{g=1}^{4} \int_{-w}^{w} \int_{-l}^{l}(-i \rho \omega) \frac{e^{-i k r} \cos \theta}{r} N_{g} d x d y V_{a m g} \\
& +\frac{1}{2 \pi} \\
& \quad \cdot \sum_{j=1}^{6} \sum_{m=1}^{N} \sum_{g=1}^{4} \int_{-w}^{w} \int_{-l}^{l} \frac{(i k r+1) e^{-i k r}}{r^{2}} \frac{\partial r}{\partial n} N_{g} d x d y P_{j m g},
\end{aligned}
$$

where $V_{\text {amg }}$ represents the normal velocity at the $g$ th node of the $m$ th element of boundary surface $S_{1} ; P_{j m g}$ is the sound pressure at the $g$ th node of the $m$ th element of boundary surface $S_{j} ; N_{g}$ is the shape function; $r=r(n, \beta)$ is the distance between node $n$ and point $\beta$, and it is replaced by the distance between node $n$ and center of element $m$ in which point $\beta$ exists, for simplicity; $\theta$ is the angle between the normal velocity $V_{a m g}$ and the distance $r$.

Hence, the sound pressure $\mathbf{P}$ at all the nodes of the boundary surface can be derived when (11) is rewritten as a matrix equation, given as

$$
\mathbf{P}=(\mathbf{I}-\mathbf{R})^{-\mathbf{1}} \mathbf{B} \mathbf{V}_{a}
$$

in which $\mathbf{I}$ is an identity matrix; $\mathbf{R}$ and $\mathbf{B}$ are defined as coefficient matrices; $\mathbf{V}_{a}$ is the nodal normal velocity vector on the boundary surface $S_{1}$, which can be represented by the vibration normal velocity vector $\mathbf{V}$ of the CLD/plate.

Finally, the sound power radiated into the cavity induced by vibration of the flexible plate is defined as

$$
\begin{aligned}
W & =\frac{1}{2} \int_{S_{1}} \operatorname{Re}\left(\mathbf{V}^{*} \mathbf{P}\right) d S_{1} \\
& =\sum_{m=1}^{N} \frac{1}{2} \int_{-l}^{l} \int_{-w}^{w} \operatorname{Re}\left(\mathbf{V}_{m}^{*} \mathbf{P}_{m}\right) d x d y \\
& =\operatorname{Re} \sum_{m=1}^{N}\left(\mathbf{V}_{m}^{*} \mathbf{D} \mathbf{P}_{m}\right),
\end{aligned}
$$

where

$$
\mathbf{D}=\frac{1}{2} \int_{-l}^{l} \int_{-w}^{w} \mathbf{N}^{T} \mathbf{N} d x d y=\frac{1}{18} w l\left[\begin{array}{cccc}
4 & 2 & 1 & 2 \\
2 & 4 & 2 & 1 \\
1 & 2 & 4 & 2 \\
2 & 1 & 2 & 4
\end{array}\right]
$$

and Re denotes the real part of the expression and superscript * denotes the complex conjugate.

\section{Optimization Problem Formulations}

3.1. Optimization Model. For flexible structures, it is not practical to have full coverage with CLD treatments for vibration or acoustic reduction due to larger added mass. Furthermore, the full coverage of CLD configurations for vibration reduction cannot warrant a minimum sound radiation. Hence, an acoustic topology optimization model taking acoustic parameters as objective function is formulated. For the acoustic cavity with CLD/plate shown in Figure 1, the sound power is selected as objective function and the topology optimization model is defined as

$$
\text { Find } \mathbf{d}=\left\{\beta_{1} \beta_{2} \cdots \beta_{n}\right\}^{T}
$$

Min W

$$
\text { Subject to } \eta=\frac{\sum_{i=1}^{n}\left(1-\beta_{i}\right)}{n} \quad\left(\beta_{i}=0,1\right) \text {. }
$$

In the model, $\mathbf{d}$ is the design variable vector; $\beta_{i}$ represents the design variable. The design variable $\beta_{i}$ can be assigned 


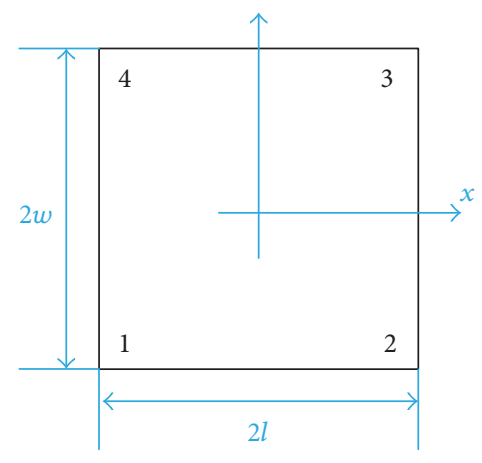

FIGURE 3: Schematic diagram of boundary element.

1 or $0 . \beta_{i}=1$ denotes that the $i$ th element is bonded with CLD material, while $\beta_{i}=0$ denotes that $i$ th element is not bonded with CLD material. $n$ denotes the elements number of CLD/plate. $\eta$ is defined as the rejection ratio of CLD material.

3.2. Acoustic Sensitivity Formulation. In topology optimization, sensitivity analysis is necessary to determine search direction. Hence, the sensitivity of objective function $W$, that is, the sound power sensitivity, with respect to design variable $\beta$, is formulated based on (13), given as follows:

$$
\frac{\partial W}{\partial \beta}=\operatorname{Re} \sum_{m=1}^{N}\left(\left(\frac{\partial \mathbf{V}_{m}^{*}}{\partial \beta}\right) \mathbf{D} \mathbf{P}_{m}+\mathbf{V}_{m}^{*} \mathbf{D}\left(\frac{\partial \mathbf{P}_{m}}{\partial \beta}\right)\right) .
$$

In order to calculate $\partial \mathbf{V}_{m}^{*} / \partial \beta$, the derivative $\partial \mathbf{X} / \partial \beta$ is derived based on (6). The partial derivative of (6) with respect to $\beta$ is carried out; this yields to

$$
\left(\frac{\partial \mathbf{K}}{\partial \beta}-\omega^{2} \frac{\partial \mathbf{M}}{\partial \beta}\right) \mathbf{X}+\left(\mathbf{K}-\omega^{2} \mathbf{M}\right) \frac{\partial \mathbf{X}}{\partial \beta}=0 .
$$

So the derivative of displacement $\mathbf{X}$ with respect to $\beta$ is formulated as

$$
\frac{\partial \mathbf{X}}{\partial \beta}=\left(\mathbf{K}-\omega^{2} \mathbf{M}\right)^{-1}\left(\frac{\partial \mathbf{K}}{\partial \beta}-\omega^{2} \frac{\partial \mathbf{M}}{\partial \beta}\right) \mathbf{X} .
$$

Hence, the velocity sensitivity of CLD/plate is induced as

$$
\frac{\partial \mathbf{V}}{\partial \beta}=-i \omega\left(\mathbf{K}-\omega^{2} \mathbf{M}\right)^{-1}\left(\frac{\partial \mathbf{K}}{\partial \beta}-\omega^{2} \frac{\partial \mathbf{M}}{\partial \beta}\right) \mathbf{X} .
$$

Furthermore, $\partial \mathbf{P} / \partial \beta$ is formulated based on (12), given as

$$
\frac{\partial \mathbf{P}}{\partial \beta}=(\mathbf{I}-\mathbf{R})^{-1} \mathbf{B}\left(\frac{\partial \mathbf{V}}{\partial \beta}\right)
$$

Substituting (19) and (20) into (16), the sensitivity of objective function $W$ can be obtained. As the design variable $\beta_{i}$ in this paper is discrete, sound radiation power sensitivity can be approximately calculated by the following expressions, given as

$$
\Delta W=\operatorname{Re} \sum_{m=1}^{N}\left(\left(\Delta \mathbf{v}_{m}^{*}\right) \mathbf{D} \mathbf{p}_{m}+\mathbf{v}_{m}^{*} \mathbf{D}\left(\Delta \mathbf{p}_{m}\right)\right) .
$$

3.3. Optimization Strategy. Evolutionary structural optimization (ESO) has been widely used to determine optimal shapes for continuum structures since Xie and Steven [16] presented this method. In this paper, it is developed to solve acoustic topology optimization problem. The advantages of ESO method lie in its simplicity in achieving shape and topology optimization for both static and dynamic problems. The flow chart of topology optimization for acoustic cavity with CLD/plate by the use of ESO is stated as follows:

(1) The number of initial removed CLD elements $R_{0}$ and evolutionary ratio ER are determined; the constraint, that is, the maximum rejection ratio of CLD material $\eta$, is set to be 0.95 in this paper.

(2) The normal vibration velocities of CLD/plate are calculated by the use of finite element method.

(3) The sound radiation power is obtained based on boundary element model of acoustic cavity with CLD/plate, considering the normal vibration velocities of CLD/plate as boundary conditions.

(4) The sensitivities of sound power are calculated.

(5) The acoustic sensitivities are modified using meshindependent filter technique [17].

(6) The modified sensitivities are sorted in descending order. Delete the $\mathrm{RR}_{0}$ elements with minimum sensitivity values.

(7) Check the constraint condition, if the constraint condition is satisfied, then stop calculation; or update $\mathrm{RR}_{0}$ by the formula of $\mathrm{RR}_{0}=\mathrm{RR}_{0}+\mathrm{ER}$

(8) Repeat procedures (3)-(7) until the constraint condition is satisfied.

The basic flowchart of the acoustic topology optimization is shown in Figure 4.

\section{Numerical Example and Discussions}

4.1. Model Configurations. Boundary surfaces of acoustic cavity are divided into $12 \times 12 \times 6$ (6 surfaces) boundary elements, and CLD/plate is also meshed into $12 \times 12$ finite elements, that is, the same as discretization of boundary surface $S_{1}$. Schematic diagram of discretization of boundary surfaces for closed cavity is shown in Figure 5. The geometrical and physical parameters for closed acoustic cavity are, unless specified otherwise, given in Table 1. For simplicity, shear modulus of damping material is expressed by complex constant modulus model, $G=G^{\infty}(1+i \eta)(\eta=0.5)$. A small structural damping is also introduced in the form of a complex elastic modulus for base plate and constrained layer: $\widetilde{E}_{i}=E_{i}\left(1+j \eta_{i}\right)(i=b, c)$, where $\eta_{i}=0.001$ is structural loss factor.

4.2. Optimal Results and Discussions. Evolutionary structural optimization (ESO) method and genetic algorithm (GA) in reference [14] are employed to search optimal CLD layouts on the flexible plate in the cavity. In the optimization process, a unit harmonic force is applied to the center of flexible plate vertically. The force is locked to the specified modal frequency of CLD/plate so that optimal CLD layouts can be obtained to minimize sound radiation power at the frequency. In this paper, the first mode frequency is considered as the first 
TABLE 1: Geometrical and physical parameters for closed acoustic cavity.

\begin{tabular}{|c|c|c|c|c|}
\hline & Young's modulus & Density & Poisson's ratio & Thickness \\
\hline Base plate & $7.0 e 4 \mathrm{MPa}$ & $2800 \mathrm{~kg} / \mathrm{m}^{3}$ & 0.3 & $0.8 \mathrm{~mm}$ \\
\hline Damping layer & $120 \mathrm{MPa}$ & $1200 \mathrm{~kg} / \mathrm{m}^{3}$ & 0.495 & $0.1 \mathrm{~mm}$ \\
\hline Constrained layer & $7.0 e 4 \mathrm{MPa}$ & $2700 \mathrm{~kg} / \mathrm{m}^{3}$ & 0.3 & $0.1 \mathrm{~mm}$ \\
\hline Cavity dimensions & \multicolumn{4}{|c|}{$0.3 \mathrm{~m} \times 0.3 \mathrm{~m} \times 0.3 \mathrm{~m}$} \\
\hline CLD/plate dimensions & \multicolumn{4}{|c|}{$0.3 \mathrm{~m} \times 0.3 \mathrm{~m}$} \\
\hline Fluid domain & \multicolumn{4}{|c|}{ Air at $25^{\circ} \mathrm{C}$ and $1 \mathrm{~atm}$} \\
\hline
\end{tabular}

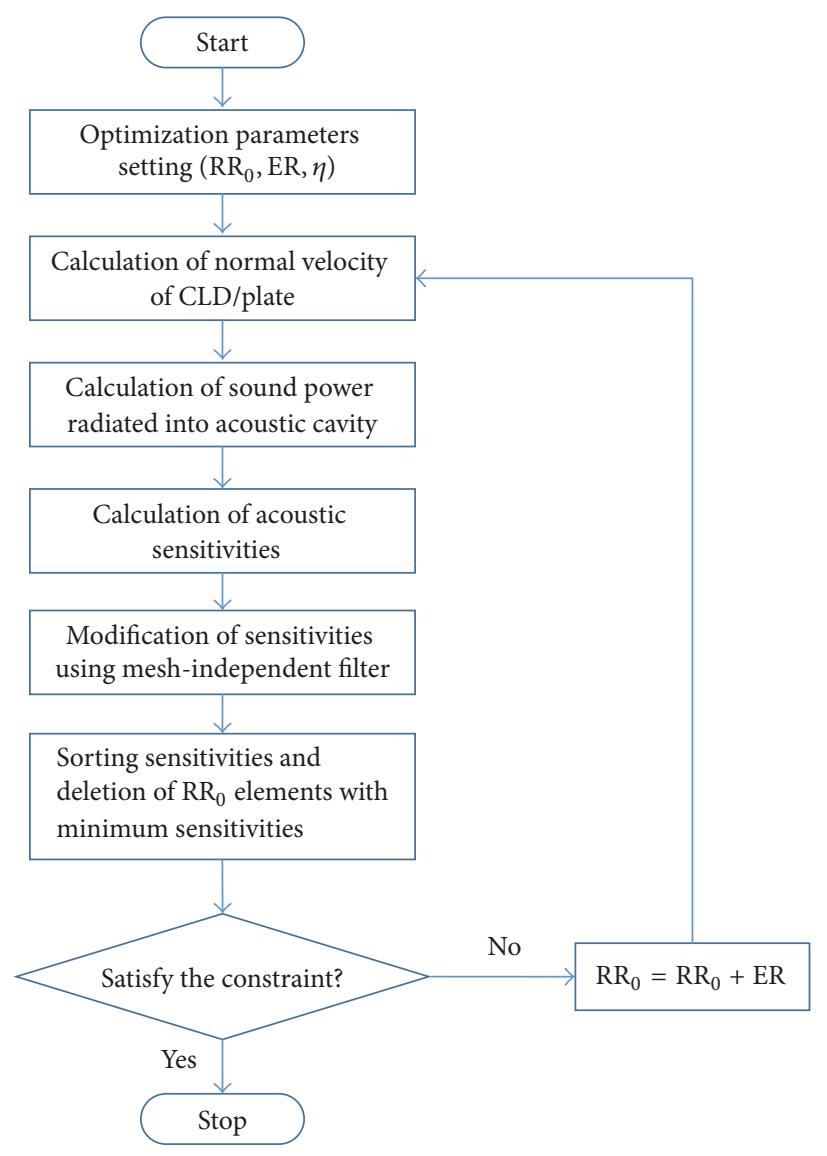

FIGURE 4: Flow chart of acoustic topology optimization using ESO.

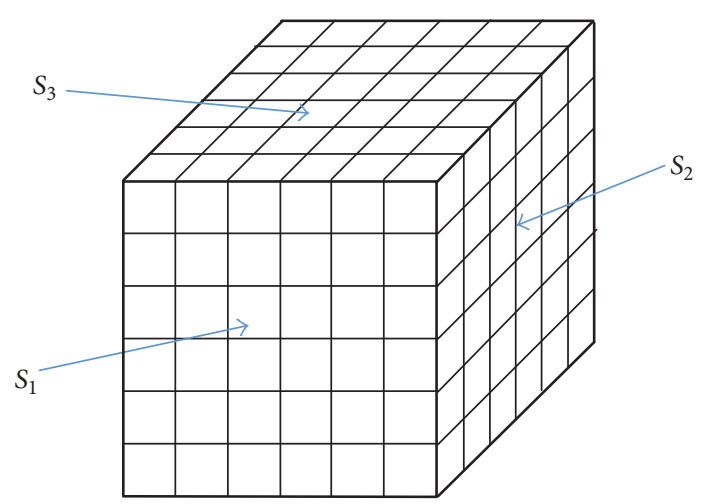

FIGURE 5: Schematic diagram of discretization of boundary surfaces for closed cavity. 


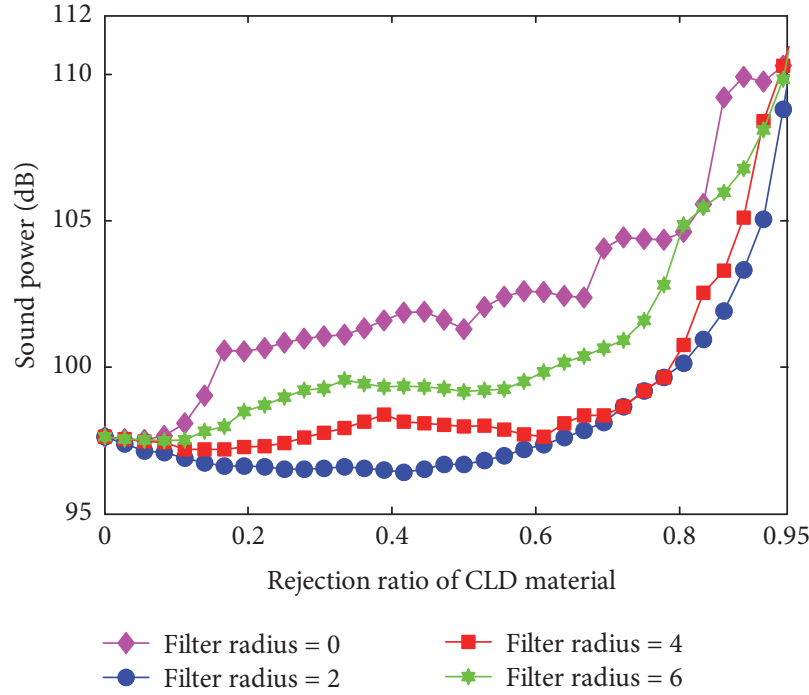

(a) The first boundary condition

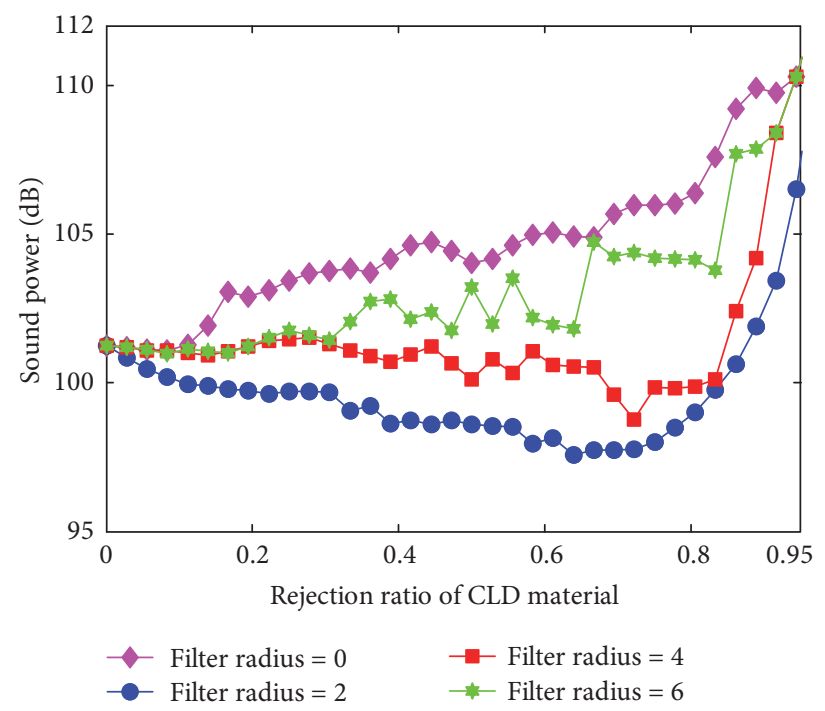

(b) The second boundary condition

FIGURE 6: Relationship between sound power and rejection ratio of CLD materials under various filter radius.

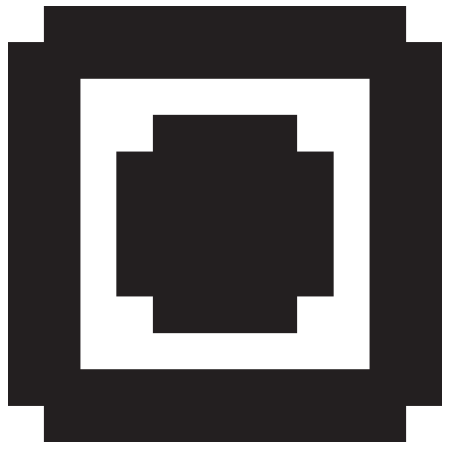

(a) Rejection ratio $=25 \%$

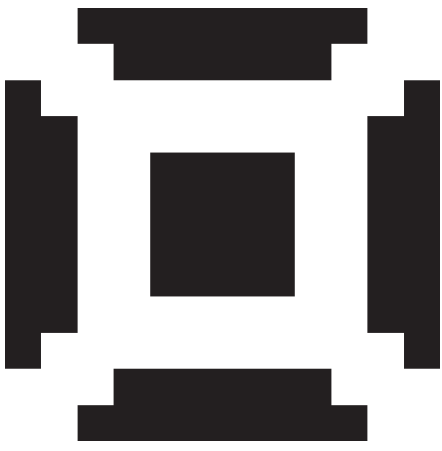

(b) Rejection ratio $=50 \%$
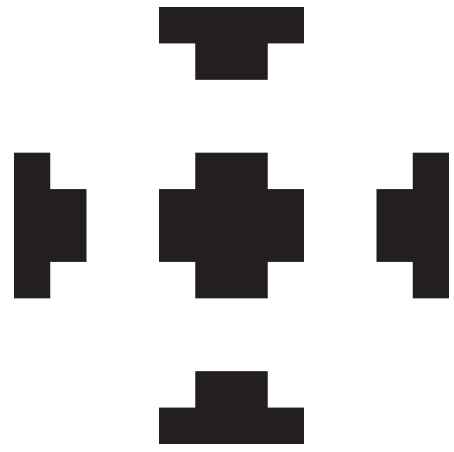

(c) Rejection ratio $=75 \%$

FIGURE 7: Optimal CLD layouts obtained using ESO under various rejection ratios for first BC.

odd mode with higher contribution for sound radiation. Due to variation of mode frequency induced by CLD materials layouts, the first mode frequency is calculated again at every iteration step and the excitation frequency is thus locked on.

All the above model and optimization process are carried out by programming Matlab code and implemented on a work station with two Intel(R) Xeon(R) CPUs (E5-2623 v3 $3.00 \mathrm{GHz}$ ) and $64.0 \mathrm{~GB}$ RAM. The finite element model and boundary element model of the CLD/plate and acoustical cavity are already validated in [14].

For CLD/plate, a common boundary condition that all the four sides of base structure are clamped is employed as the first BC. Here, another boundary condition, four sides of base plate are clamped and the sides of CLD treatments on the plate edges are proposed as the second $\mathrm{BC}$ for $\mathrm{CLD} /$ plate.

\subsubsection{Optimal Results Obtained Using ESO. In optimization} process using ESO, evolution parameters $\mathrm{RR}_{0}$ and ER are set to be 0 and 4, respectively. Filter radius in mesh-independent filter technique is a key parameter and the influences of various filter radius on optimal results are analyzed, given in Figure 6. It is clear that while filter radius is equal to 2, the larger sound power reduction can be obtained for the above two boundary conditions. So filter radius equal to 2 is assigned in the following optimization process.

Figures 7 and 8 present optimal layouts of CLD treatments on flexible plate with the two BCs under various rejection ratios for the first mode. It is very clear that CLD treatments layouts are very regular and boundary conditions have significant effect on the optimal CLD layouts.

The frequency responses of sound radiation power are also monitored. Figures 9(a) and 9(b) are the comparisons on sound radiation power of full coverage, topology optimized with various rejections ratios, and base plate. It can be seen that sound power of the plate treated with optimal CLD layouts is reduced significantly compared with that of the base plate, in which the maximum reduction of sound power is achieved as $26.9 \mathrm{~dB}$ and $25.4 \mathrm{~dB}$ for first $\mathrm{BC}$ and second BC, respectively. An interesting result can be found in Figure 9(a); that is, for the plate with full coverage of CLD materials, 


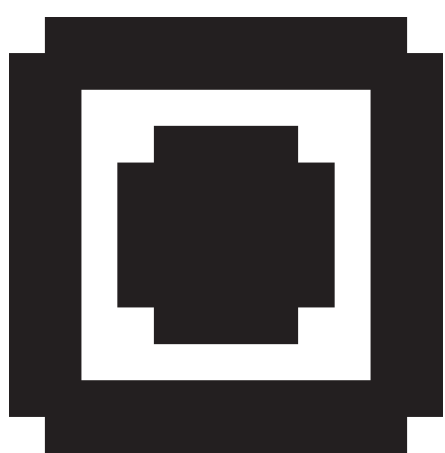

(a) Rejection ratio $=25 \%$

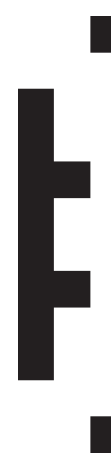

(b) Rejection ratio $=50 \%$

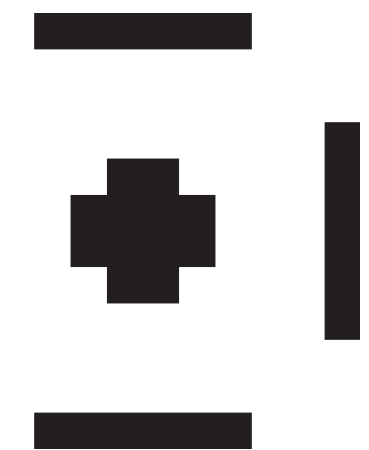

(c) Rejection ratio $=75 \%$

FIGURE 8: Optimal CLD layouts obtained using ESO under different rejection ratios for second BC.

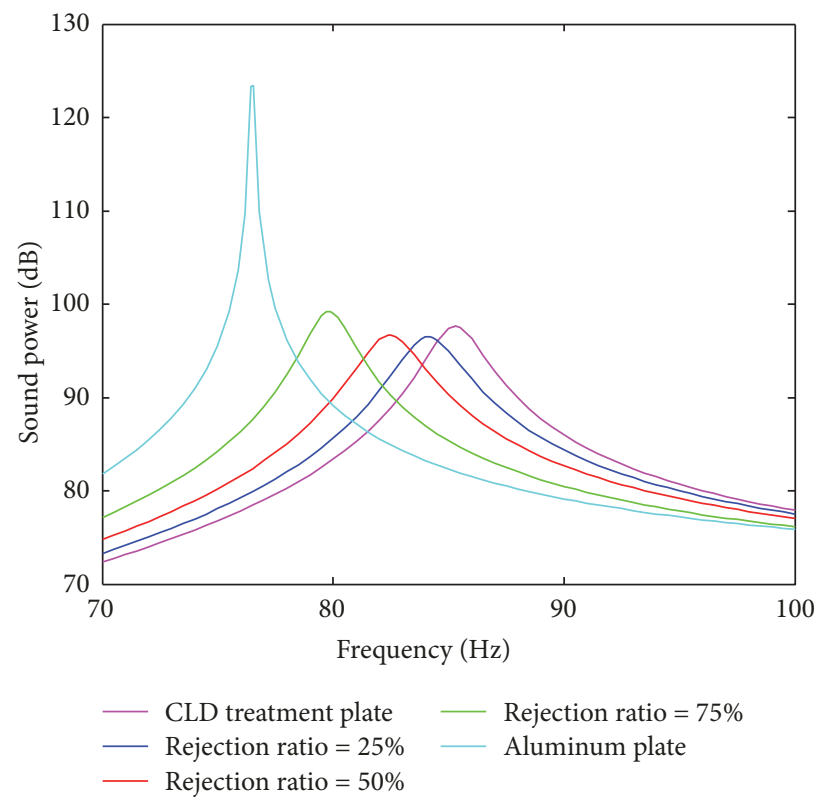

(a) The first boundary condition

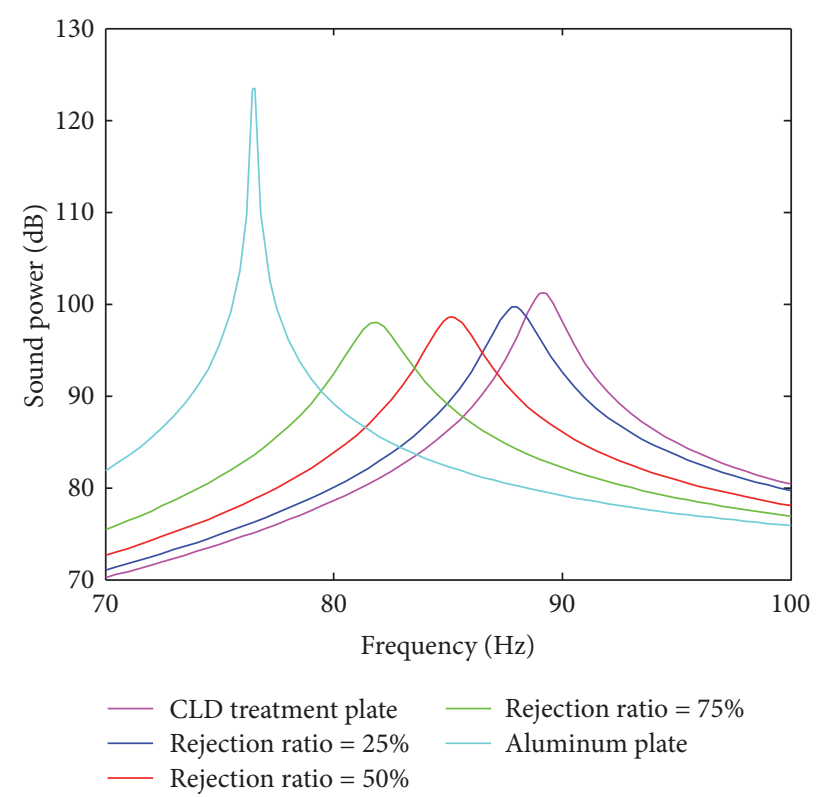

(b) The second boundary condition

FIGURE 9: Frequency responses of sound power obtained using ESO under various rejection ratios.

the sound power reduction is about $25.8 \mathrm{~dB}$, even slightly smaller than that for $75 \%$ coverage (rejection ratio $=25 \%$, $26.9 \mathrm{~dB}$ ) and $50 \%$ coverage (rejection ratio $=50 \%, 26.7 \mathrm{~dB}$ ). A similar result can be obtained from Figure 9(b). That indicates a higher CLD coverage does not guarantee better sound radiation reduction. Furthermore, a comparison on sound power with optimal CLD layouts at different rejection ratios is depicted in Figure 10 for first BC and second BC. It can be observed that there is an optimal rejection ratio (point 1 and point 2) which makes highest sound power reduction. For the first boundary condition, the higher sound power reduction can be obtained with comparison to that of the second boundary condition while the rejection ratio is less than $63.9 \%$. Otherwise, the larger sound power reduction for the second boundary condition is achieved.

4.2.2. Optimal Results Obtained Using GA. For comparison purposes, a genetic algorithm with integer encoding in
[14] is also used to search optimal layouts of CLD/plate to minimize sound power for first mode. The locations number of CLD treatments is initialized in a random integer vector as individuals (design variables) in GA. Due to symmetry of the flexible plate, nine and eighteen variables are defined using locations number of CLD material for $25 \%$ and $50 \%$ rejection ratios and nine variables are defined using locations number at which the CLD materials are not bonded for $75 \%$ rejection ratios. As an example shown in Figure 11 , for $25 \%, 50 \%$, and $75 \%$ rejection ratios, the individuals are constructed as $[1,2,3,10,17,20,23,32,33]$, [2, $4,9,10,12,13,14,15,16,20,22,23,25,28,29,30,32,34]$, and $[1,3,6,15,21,24,26,31,36]$, respectively.

In optimization process, other parameters are the same as that in [14]; that is, the crossover probability and the mutation probability are set to be 0.9 and 0.1 , respectively, and the genetic parameters $b$ and $p$ are set to be 0.25 and 10 , respectively. The population size $N_{\text {pop }}$ of GA is set to be 100 , 


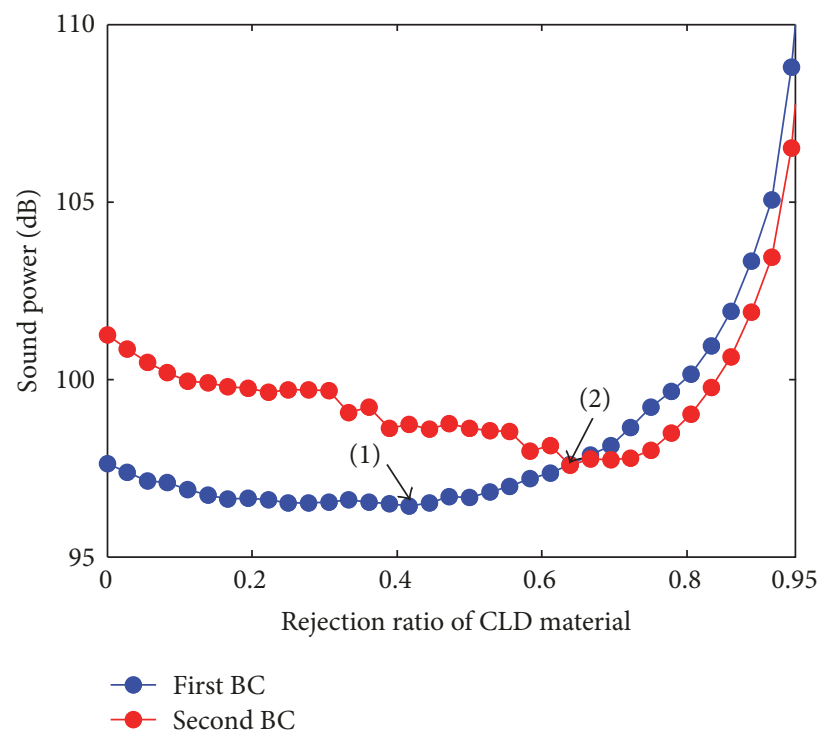

FIGURE 10: The relationship between sound power and rejection ratios of CLD materials obtained using ESO.

\begin{tabular}{|c|c|c|c|c|c|}
\hline 1 & 7 & 13 & 19 & 25 & 31 \\
\hline 2 & 8 & 14 & 20 & 26 & 32 \\
\cline { 2 - 5 } 3 & 9 & 15 & 21 & 27 & 33 \\
\hline 4 & 10 & 16 & 22 & 28 & 34 \\
\hline 5 & 11 & 17 & 23 & 29 & 35 \\
\hline 6 & 12 & 18 & 24 & 30 & 36 \\
\hline
\end{tabular}

(a) Rejection ratio $=25 \%$

\begin{tabular}{|c|c|c|c|c|c|}
\hline 1 & 7 & 13 & 19 & 25 & 31 \\
\hline 2 & 8 & 14 & 20 & 26 & 32 \\
\hline 3 & 9 & 15 & 21 & 27 & 33 \\
\hline 4 & 10 & 16 & 22 & 28 & 34 \\
\hline 5 & 11 & 17 & 23 & 29 & 35 \\
\hline 6 & 12 & 18 & 24 & 30 & 36 \\
\hline
\end{tabular}

(b) Rejection ratio $=50 \%$

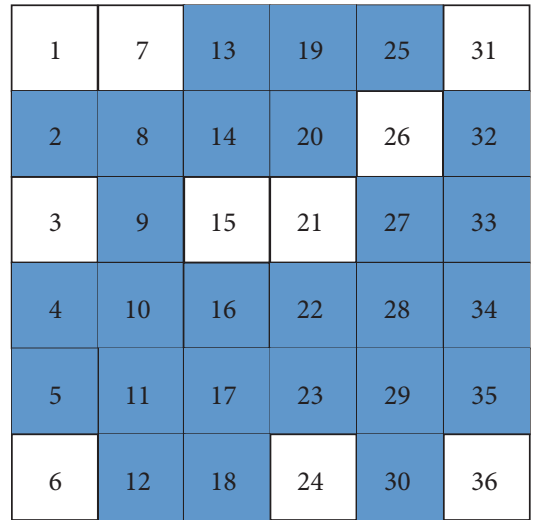

(c) Rejection ratio $=75 \%$

FIGURE 11: Schematic diagram of design variables in GA optimization process.

and the maximum evolutionary generation is also set to be 100. While evolutionary generations achieve maximum number of generations or optimal solution holds the same value for continuous fifteen generations, the evolution process is terminated.

Optimization process is simulated ten times for every above rejection ratio and the best solutions are selected. The corresponding layouts of CLD treatments with the above two BCs for first mode are presented in Figures 12 and 13. The corresponding frequency responses of sound power are shown in Figure 14. It is very apparent that optimal CLD materials layouts become notably different with $25 \%$ and $50 \%$ rejection ratios due to different $\mathrm{BCs}$, but the same layouts can be obtained at $75 \%$ rejection ratio. Similar results to that from Figure 9 can be obtained from Figure 14.

4.2.3. Comparison and Discussions. The minimum sound power for the two BCs obtained using ESO and GA is listed in Table 2. It is found that sound power obtained using GA is slightly smaller than that obtained using ESO except for $75 \%$ rejection ratio for second $\mathrm{BC}$. That is induced by the difference of optimization mechanism of ESO and GA. For ESO, several CLD elements are deleted according to sorting sound power sensitivities at every iteration, and the sensitivities are in coupling between the two adjacent iterations. Hence, the relationship between sound power and rejection ratios can be monitored and optimal rejection ratio can be found, as an example, shown in Figure 10. For GA, the number of added CLD elements is a constant value while a rejection ratio is defined, and optimal locations of CLD elements are searched according to sorting the sound power directly in evolutionary process.

The total execution time $t_{1}$ and the average execution time $t_{a}$ per iteration for ESO are listed in Table 3 under different rejection ratios. It can be observed that the average execution time decreases with rejection ratios increasing. 


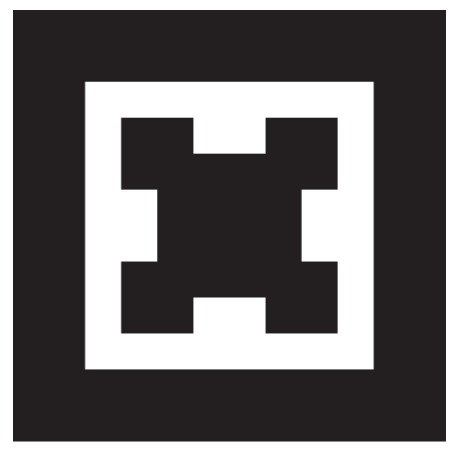

(a) Rejection ratio $=25 \%$

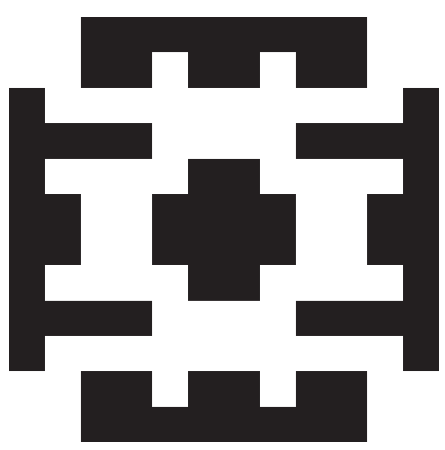

(b) Rejection ratio $=50 \%$

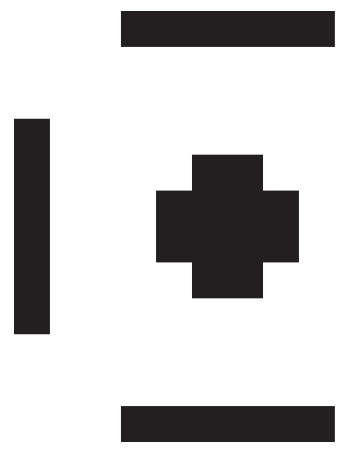

(c) Rejection ratio $=75 \%$

FIGURE 12: Optimal CLD layouts obtained using GA under various rejection ratios for first BC.

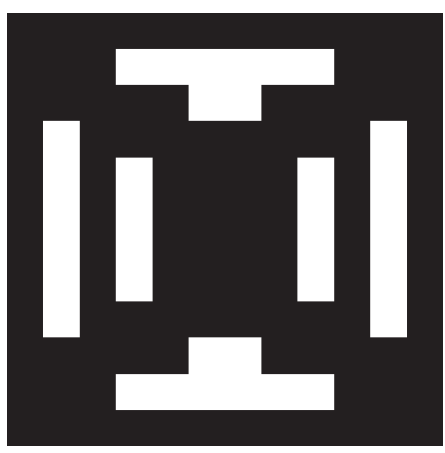

(a) Rejection ratio $=25 \%$

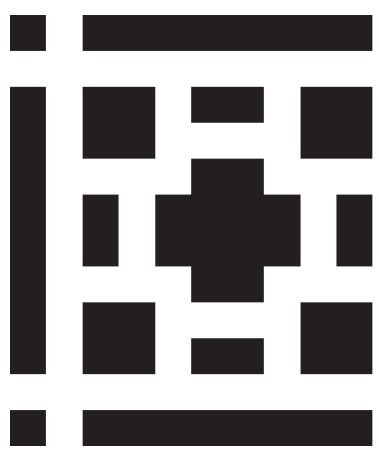

(b) Rejection ratio $=50 \%$
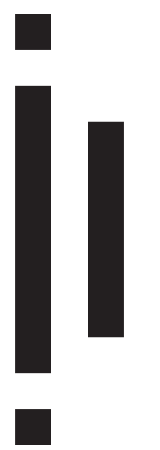

(c) Rejection ratio $=75 \%$

FIGURE 13: Optimal CLD layouts obtained using GA under various rejection ratios for second BC.

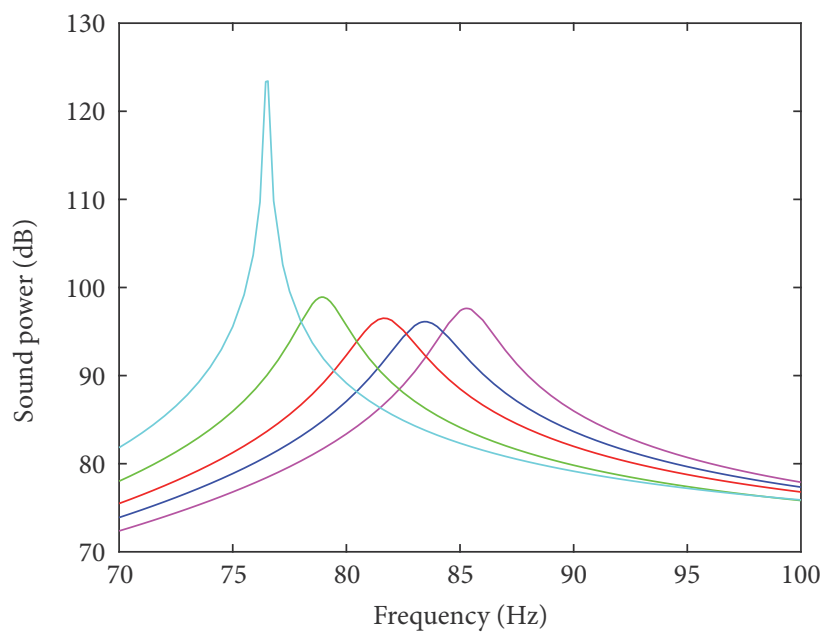

- CLD treatment plate - Rejection ratio $=75 \%$

- Rejection ratio $=25 \%$

Rejection ratio $=50 \%$

(a) The first boundary condition

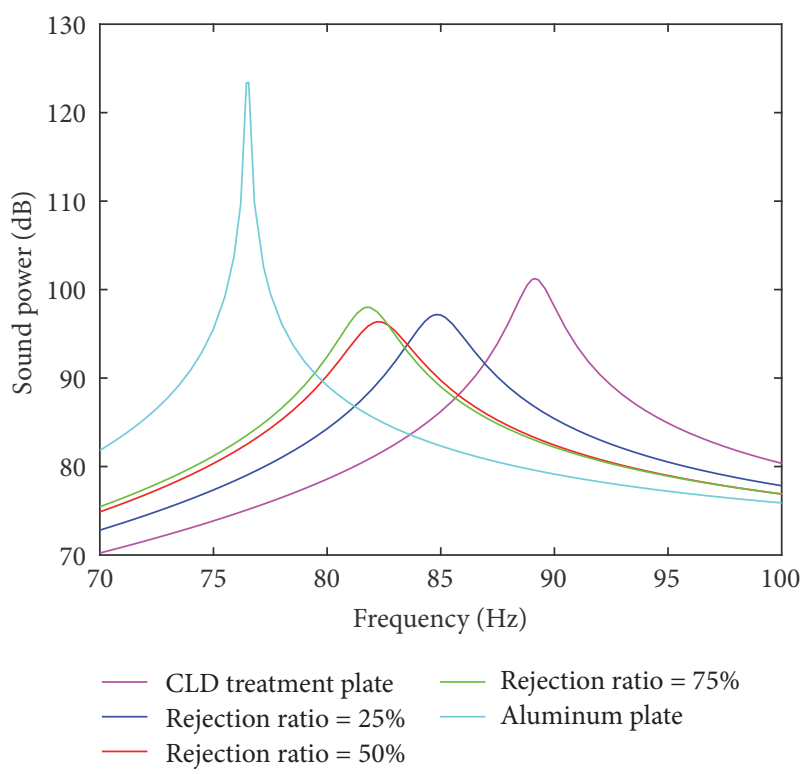

(b) The second boundary condition

FIGURE 14: Frequency responses of sound power under various rejection ratios obtained using GA. 
TABLE 2: Minimum sound power obtained using of ESO and GA (dB).

\begin{tabular}{|c|c|c|c|c|}
\hline \multirow{2}{*}{ Rejection ratio } & \multicolumn{2}{|c|}{ ESO } & \multicolumn{2}{|c|}{ GA } \\
\hline & First BC & Second BC & First BC & Second BC \\
\hline $25 \%$ & $96.51 \mathrm{~dB}$ & $99.71 \mathrm{~dB}$ & $96.12 \mathrm{~dB}$ & $97.18 \mathrm{~dB}$ \\
\hline $50 \%$ & $96.67 \mathrm{~dB}$ & $98.61 \mathrm{~dB}$ & $96.51 \mathrm{~dB}$ & $96.37 \mathrm{~dB}$ \\
\hline $75 \%$ & $99.21 \mathrm{~dB}$ & $98.00 \mathrm{~dB}$ & $98.91 \mathrm{~dB}$ & $98.00 \mathrm{~dB}$ \\
\hline
\end{tabular}

TABle 3: Execution time in seconds using ESO.

\begin{tabular}{|c|c|c|c|c|c|c|}
\hline \multirow{2}{*}{ Rejection ratio } & \multicolumn{2}{|c|}{$25 \%$} & \multicolumn{2}{|c|}{$50 \%$} & \multicolumn{2}{|c|}{$75 \%$} \\
\hline & $t_{1}$ & $t_{a}$ & $t_{1}$ & $t_{a}$ & $t_{1}$ & $t_{a}$ \\
\hline First BC & 403 & 44.8 & 739 & 41.0 & 1036 & 38.4 \\
\hline Second BC & 355 & 39.4 & 659 & 36.6 & 945 & 35.0 \\
\hline
\end{tabular}

TABLE 4: Execution time in seconds using GA for first BC.

\begin{tabular}{|c|c|c|c|c|c|c|c|c|c|}
\hline \multirow{2}{*}{$\begin{array}{l}\text { Simulation } \\
\text { number }\end{array}$} & \multicolumn{3}{|c|}{ Rejection ratio $=25 \%$} & \multicolumn{3}{|c|}{ Rejection ratio $=50 \%$} & \multicolumn{3}{|c|}{ Rejection ratio $=75 \%$} \\
\hline & SPL/dB & $t_{1}$ & $t_{a}$ & $\mathrm{SPL} / \mathrm{dB}$ & $t_{1}$ & $t_{a}$ & $\mathrm{SPL} / \mathrm{dB}$ & $t_{1}$ & $t_{a}$ \\
\hline (1) & $96.16 \mathrm{~dB}$ & 6552 & $384 \mathrm{~s}$ & 96.67 & 5634 & 421 & 99.03 & 4910 & 327 \\
\hline (2) & $96.29 \mathrm{~dB}$ & 8392 & $378 \mathrm{~s}$ & 96.75 & 7998 & 409 & 99.03 & 5989 & 323 \\
\hline (3) & $96.27 \mathrm{~dB}$ & 6556 & $385 \mathrm{~s}$ & 96.67 & 10372 & 401 & 98.91 & 5047 & 330 \\
\hline (4) & $96.27 \mathrm{~dB}$ & 6502 & $382 \mathrm{~s}$ & 96.87 & 8377 & 412 & 98.91 & 4606 & 340 \\
\hline (5) & $96.27 \mathrm{~dB}$ & 4055 & $338 \mathrm{~s}$ & 96.63 & 8919 & 414 & 99.14 & 3630 & 338 \\
\hline (6) & $96.33 \mathrm{~dB}$ & 7208 & 392 & 96.79 & 8022 & 411 & 99.68 & 5938 & 328 \\
\hline (7) & $96.25 \mathrm{~dB}$ & 6744 & 390 & 96.51 & 9346 & 413 & 99.02 & 5601 & 328 \\
\hline (8) & $96.12 \mathrm{~dB}$ & 5644 & 398 & 96.64 & 7879 & 404 & 99.03 & 7257 & 324 \\
\hline (9) & $96.26 \mathrm{~dB}$ & 5643 & 399 & 96.66 & 12473 & 407 & 98.91 & 6604 & 326 \\
\hline$(10)$ & $96.27 \mathrm{~dB}$ & 9561 & 376 & 96.60 & 7099 & 408 & 99.03 & 4548 & 325 \\
\hline
\end{tabular}

TABLE 5: Execution time in seconds using GA for second BC.

\begin{tabular}{|c|c|c|c|c|c|c|c|c|c|}
\hline \multirow{2}{*}{$\begin{array}{l}\text { Simulation } \\
\text { number }\end{array}$} & \multicolumn{3}{|c|}{ Rejection ratio $=25 \%$} & \multicolumn{3}{|c|}{ Rejection ratio $=50 \%$} & \multicolumn{3}{|c|}{ Rejection ratio $=75 \%$} \\
\hline & $\mathrm{SPL} / \mathrm{dB}$ & $t_{1}$ & $t_{a}$ & $\mathrm{SPL} / \mathrm{dB}$ & $t_{1}$ & $t_{a}$ & $\mathrm{SPL} / \mathrm{dB}$ & $t_{1}$ & $t_{a}$ \\
\hline (1) & 97.181 & 4962 & 327 & 96.60 & 8758 & 357 & 98.00 & 5194 & 321 \\
\hline (2) & 92.227 & 5587 & 330 & 96.58 & 7267 & 358 & 98.62 & 1253 & 328 \\
\hline (3) & 97.181 & 4091 & 334 & 96.72 & 5729 & 345 & 98.20 & 4924 & 322 \\
\hline (4) & 97.180 & 3074 & 329 & 96.72 & 5467 & 338 & 98.16 & 10096 & 302 \\
\hline (5) & 97.180 & 5914 & 332 & 96.72 & 5588 & 340 & 98.27 & 5545 & 322 \\
\hline (6) & 97.180 & 4133 & 337 & 96.47 & 11385 & 346 & 98.00 & 7820 & 304 \\
\hline (7) & 97.181 & 7638 & 330 & 96.37 & 9298 & 344 & 98.16 & 3809 & 310 \\
\hline (8) & 97.180 & 4375 & 332 & 96.58 & 7024 & 351 & 98.00 & 4658 & 308 \\
\hline (9) & 97.316 & 4701 & 325 & 96.58 & 7537 & 351 & 98.51 & 3476 & 310 \\
\hline$(10)$ & 97.180 & 6372 & 321 & 96.61 & 5812 & 341 & 98.44 & 3777 & 309 \\
\hline
\end{tabular}

That is because the total useful degrees of freedom decrease with CLD elements being deleted. It is also noted that the best solutions are deterministic for ESO while the parameters of ESO are assigned.

The minimum sound power, the execution time $t_{1}$, at which the minimum sound power is found firstly, and the average execution time $t_{a}$ per iteration for GA are listed in Tables 4 and 5. From the two tables, the minimum sound power in bold corresponding to selected optimal layouts in Figures 12 and 13 can be observed. It can be seen that best solutions are not always obtained by one-time execution of optimization process for GA. Meanwhile, it requires more 
TABLE 6: Consistency ratios of optimal CLD layouts using ESO and GA.

\begin{tabular}{llll}
\hline Rejection ratio & $25 \%$ & $50 \%$ & $75 \%$ \\
\hline First BC & 0.93 & 0.83 & 0.78 \\
Second BC & 0.67 & 0.61 & 1.00 \\
\hline
\end{tabular}

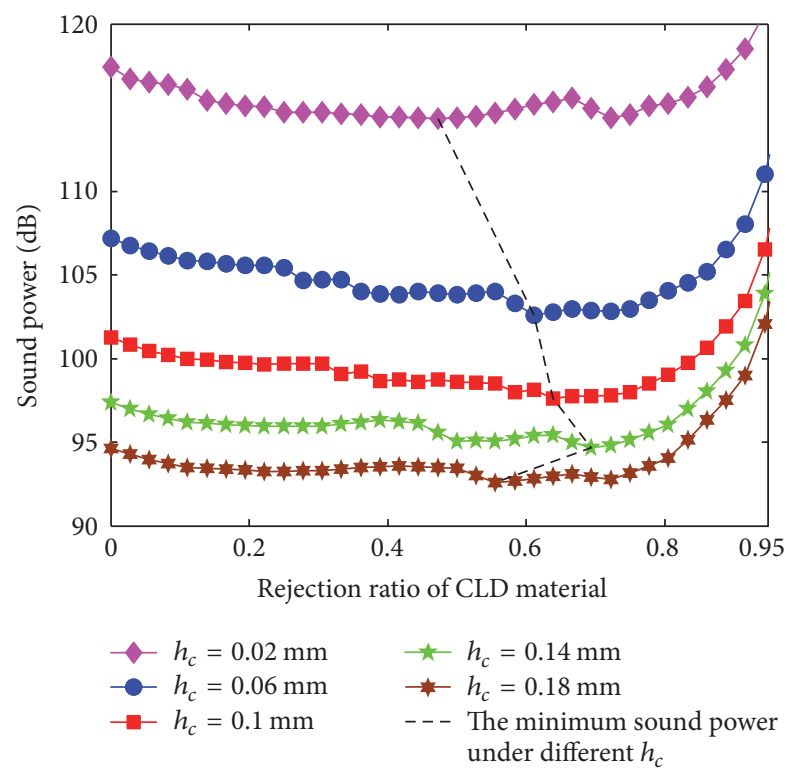

(a) The first boundary condition

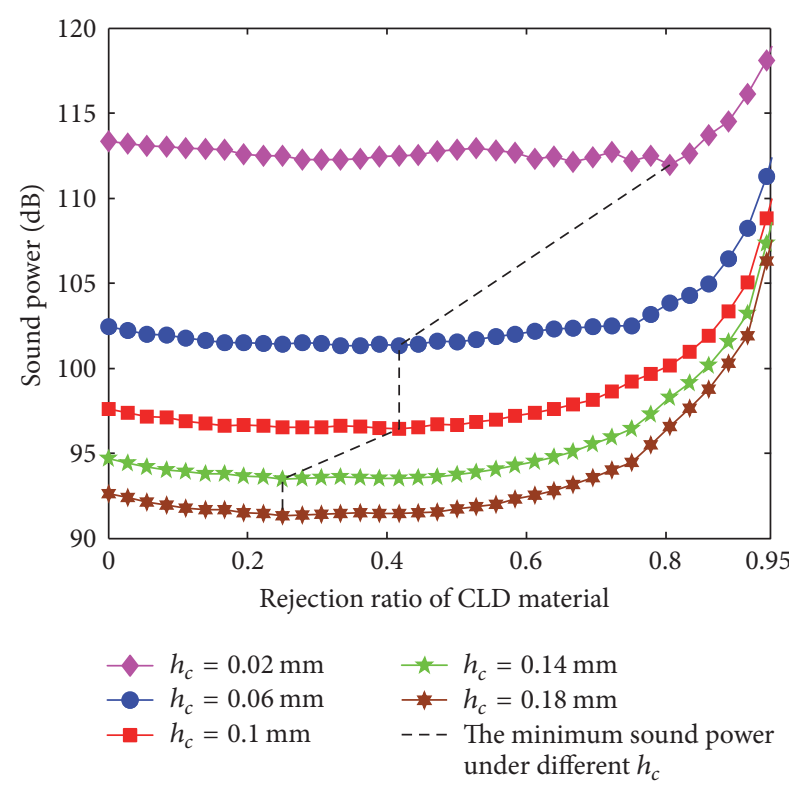

(b) The second boundary condition

FIGURE 15: Influence for sound power with rejection ratios under various $h_{c}$.

computational time to find the best solution compared to ESO. While the number of design variables is smaller, the rate to find best solution is higher in the ten times simulations.

Further, a consistency ratio is defined to describe the relativity of optimal CLD treatments layouts obtained using ESO and GA, that is, a ratio of total number of the same locations of CLD treatments to total number of CLD treatments under a rejection ratio, seen in Table 6 . For first $\mathrm{BC}$, there is a better consistency than that of second $\mathrm{BC}$, and it can be also inferred that the same optimal CLD treatments layout can be obtained using ESO and GA at a smaller rejection ratio. From comparison between optimal results, optimal CLD layouts obtained using ESO are always regular, but optimal CLD layouts obtained using GA are not always irregular and centrosymmetric. That implies mesh-independence phenomenon exists in the optimization process using GA, which makes the optimal layouts not easy to realize in engineering applications.

Integrating the above comparison and analysis, it can be concluded that the ESO is more efficient to obtain deterministic and more practical optimal CLD material layouts for minimizing sound radiation power compared to GA.

\subsection{Discussions of Optimal Results Obtained Using ESO under Different Key Parameters}

4.3.1. Discussions of Optimal Results with Different $h_{c}$ and $h_{v}$. In topology optimization study for CLD structures, the positions of CLD materials are usually considered as design variables while a specific thickness of CLD materials is defined, such as that in $[8,9,11,14,17]$. To the authors' knowledge, the influences on topology optimization results induced by the thickness of CLD materials are quite less. Hence, the influence of $h_{c}$ and $h_{v}$ on minimum sound radiation power obtained using ESO is discussed. Figures 15 and 16 show variation of sound radiation power with $h_{c}$ and $h_{v}$ increasing for first $\mathrm{BC}$ and second $\mathrm{BC}$ when rejection ratios increase. Figures 15 and 16 illustrate the following: (1) While $h_{c}$ and $h_{v}$ increase, larger sound power reduction can be achieved. (2) There is always an optimal rejection ratio (an optimal coverage ratio) which makes maximum sound power reduction for every setting of $h_{c}$ and $h_{v}$. (3) When $h_{c}$ increases, the optimal rejection ratio increases firstly and then decrease for the first $\mathrm{BC}$, contrary to the decreasing trend for the second BC. (4) When $h_{v}$ increases, the optimal rejection ratio increases for the first $\mathrm{BC}$, but the optimal rejection ratio decreases for the second $\mathrm{BC}$.

4.3.2. Discussions of Optimal Results with Different Locations of Exciting Force. The influence of exciting force locations on optimal CLD materials layouts is also discussed. Figure 17 illustrates the finite element partition of $1 / 4 \mathrm{CLD} /$ plate in the closed cavity, in which point $C$ (center of the plate), point 1 , point 2 , point 3 , and point 4 are locations of applied unit exciting force. Figures 18 and 19 show optimal CLD materials layouts under different rejection ratios while the plate is 


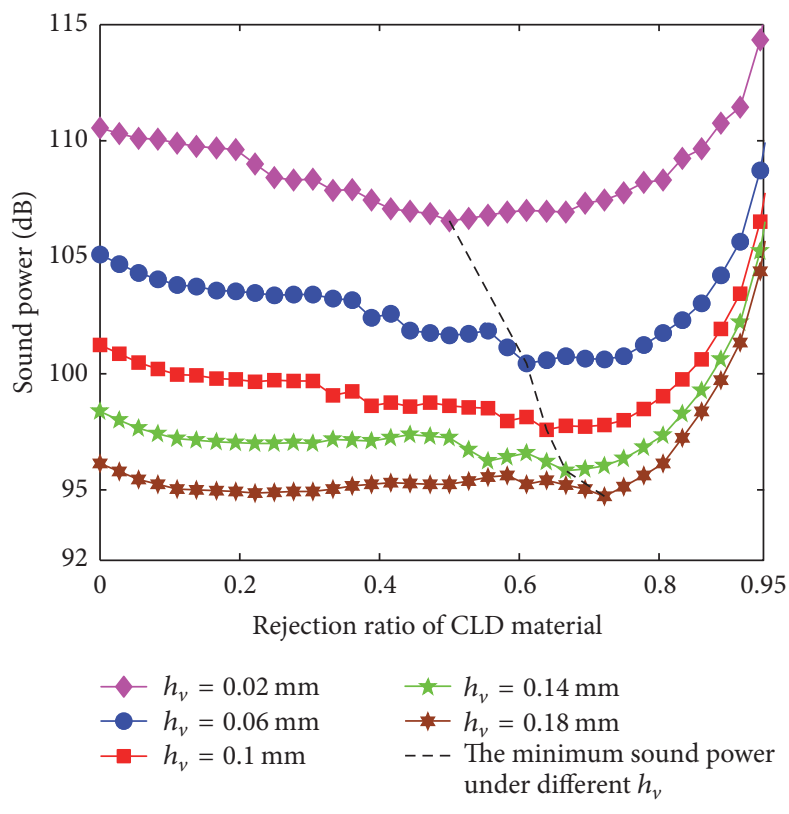

(a) The first boundary condition

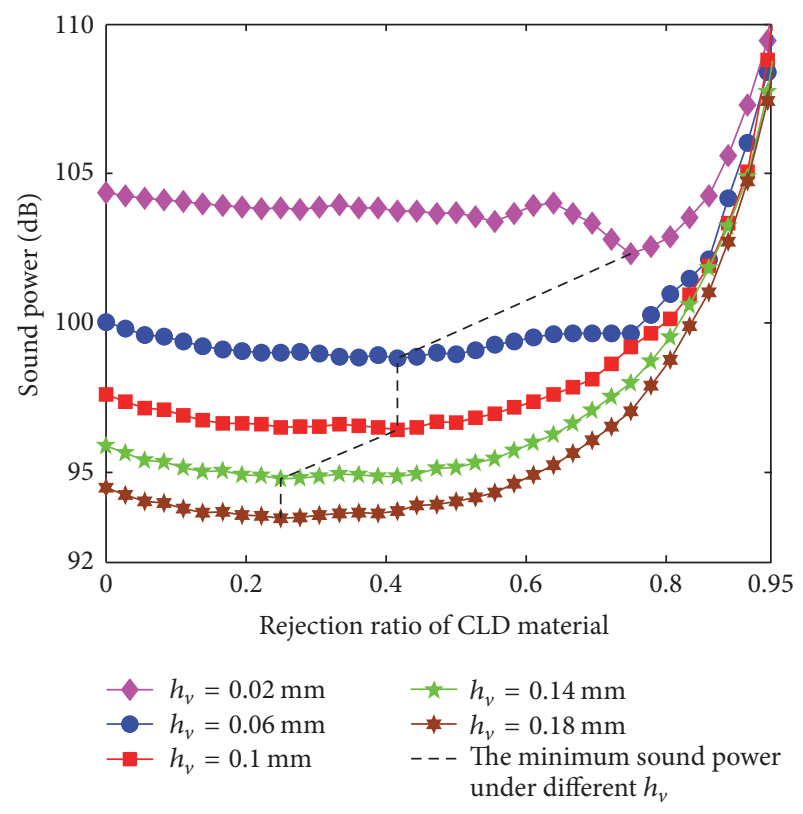

(b) The second boundary condition

FIGURE 16: Influence for sound power with rejection ratio under various $h_{v}$.

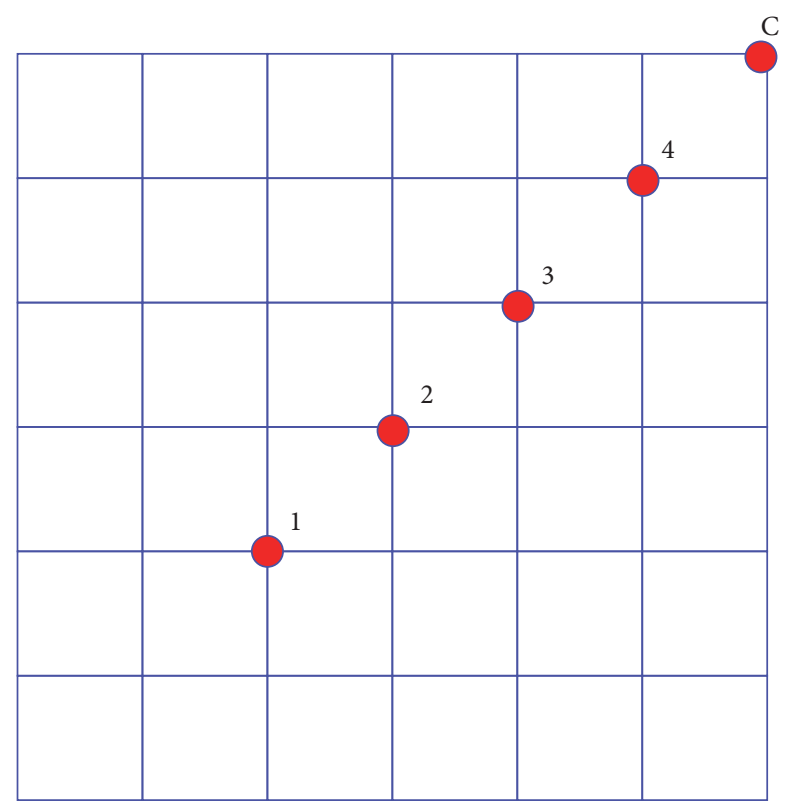

FIGURE 17: Locations of exciting force shown at finite element partition of $1 / 4 \mathrm{CLD} /$ plate.

excited at point 1 for first BC and second BC. For simplicity, only three different rejection ratios are given. Comparing Figure 7 with Figure 18, it is apparent that variation of exciting force locations makes optimal CLD layouts at the same rejection ratio be changed. However, when the rejection ratio increases to a special value, optimal layouts of CLD materials will not be changed with variation of locations of exciting force. Similar results can be obtained from a comparison of Figures 8 and 19. It can be inferred that the above results are induced by the difference of vibration normal velocity distribution of original structure in optimization process. The vibration normal velocity distribution is given in Figures 20 and 21.

The best rejection ratios with varied locations of exciting force are also monitored, given in Table 7. It can be concluded that, for first BC, the plate is covered with about $60 \%$ $70 \%$ CLD materials; better sound power reduction can be achieved; for second $\mathrm{BC}$, this is about $35 \% \sim 45 \%$. 


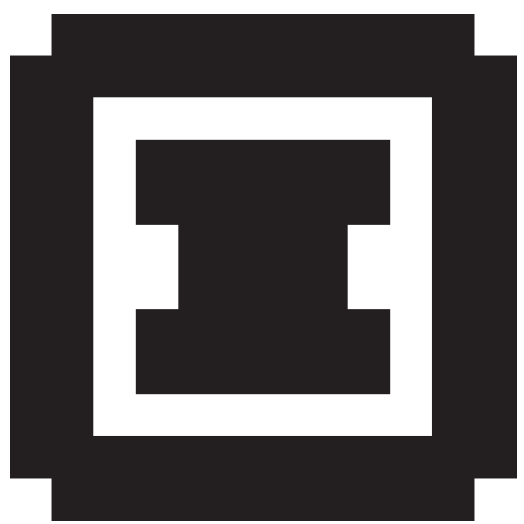

(a) Rejection ratio $=25 \%$

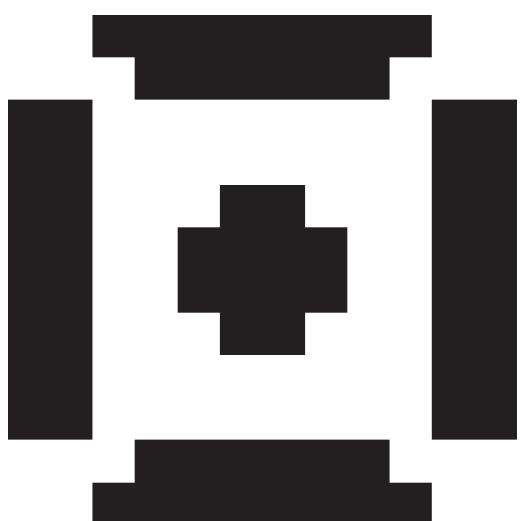

(b) Rejection ratio $=50 \%$
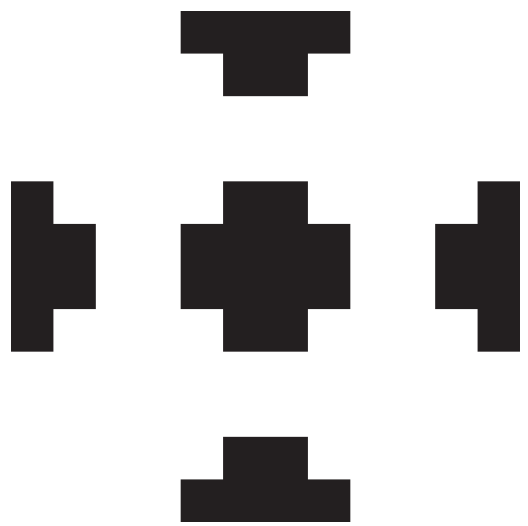

(c) Rejection ratio $=75 \%$

FIGURE 18: Optimal layouts of CLD treatments under exciting force at point 1 for first BC.

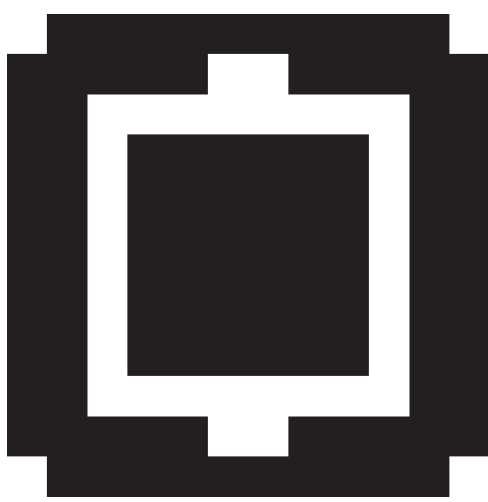

(a) Rejection ratio $=25 \%$

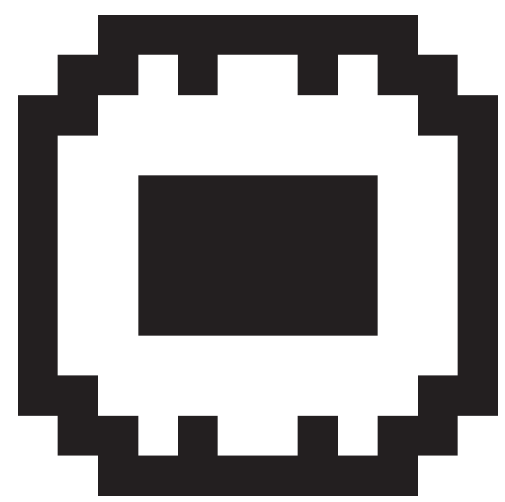

(b) Rejection ratio $=50 \%$

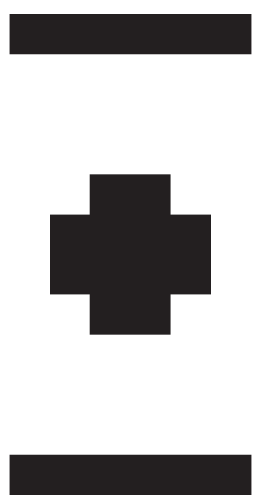

(c) Rejection ratio $=75 \%$

FIGURE 19: Optimal layouts of CLD treatments under exciting force at point 1 for second BC.

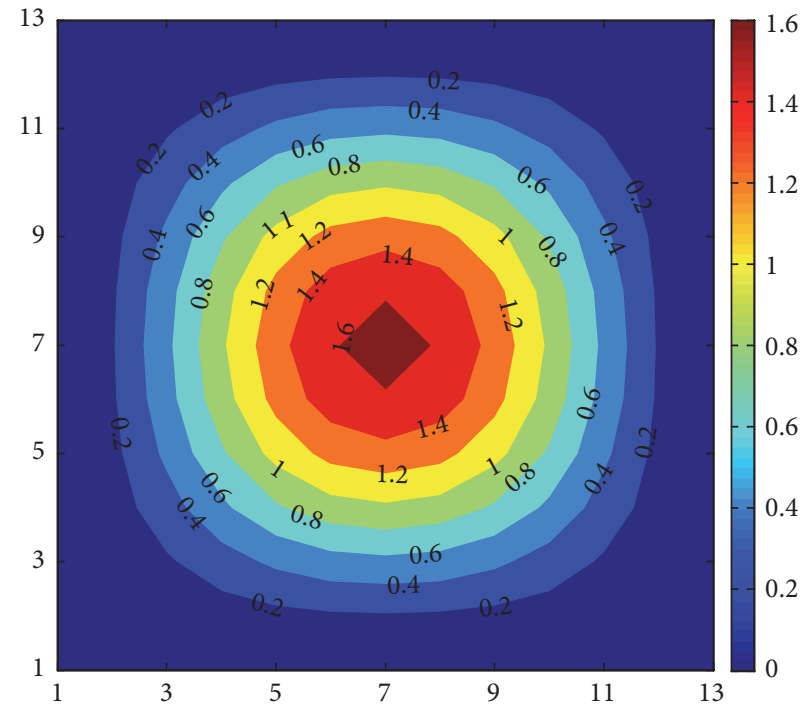

(a) The force is applied at point $\mathrm{C}$

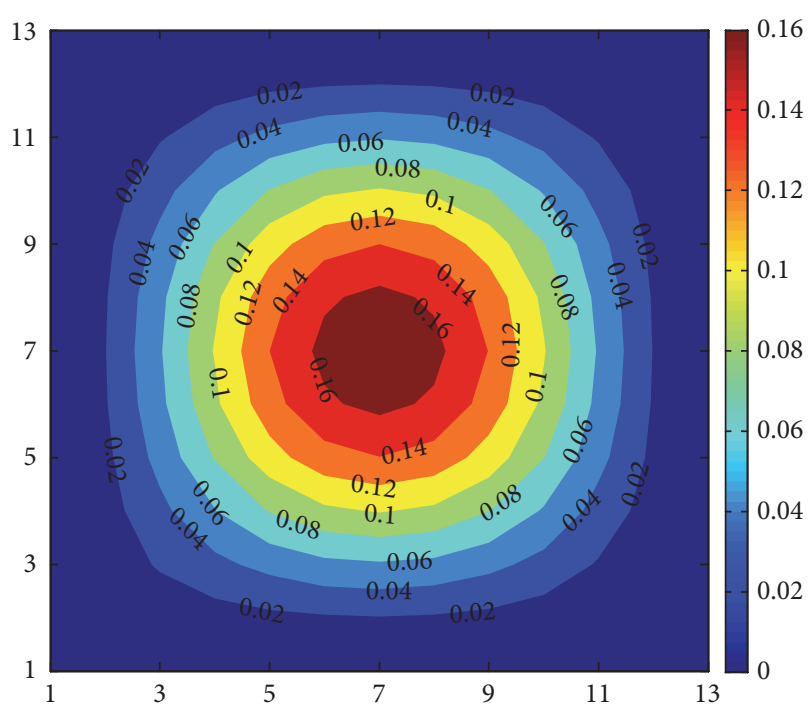

(b) The force is applied at point 1

FIGURE 20: The vibration normal velocity in the full coverage plate with CLD materials for first BC. 
TABLE 7: The best rejection ratios with varied locations of exciting force.

\begin{tabular}{lccccc}
\hline & Point 1 & Point 2 & Point 3 & Point 4 & Point C \\
\hline First BC & $27.8 \%$ & $33.3 \%$ & $38.9 \%$ & $30.6 \%$ & $41.7 \%$ \\
Second BC & $55.6 \%$ & $52.8 \%$ & $61.1 \%$ & $66.7 \%$ & $63.9 \%$ \\
\hline
\end{tabular}

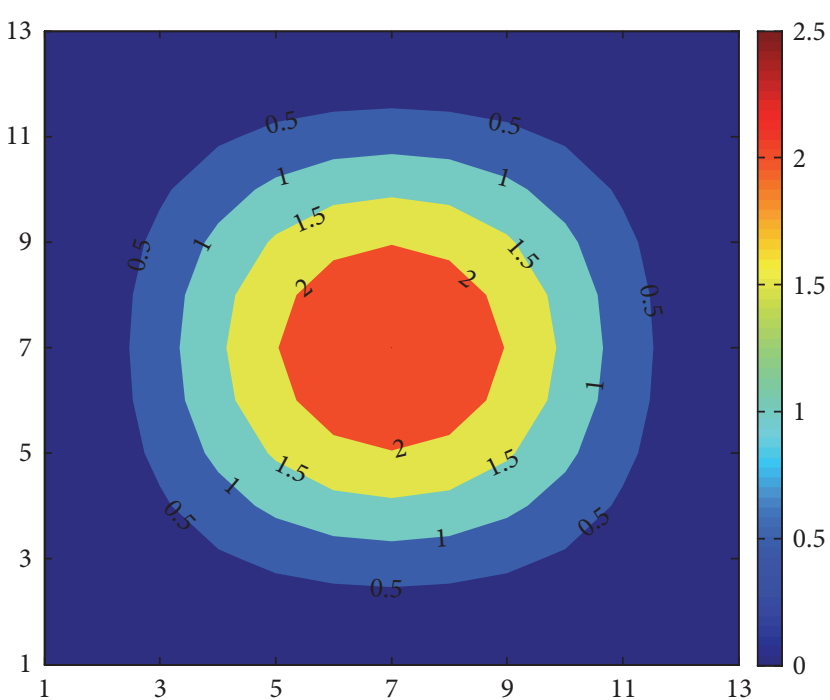

(a) The force is applied at point $\mathrm{C}$

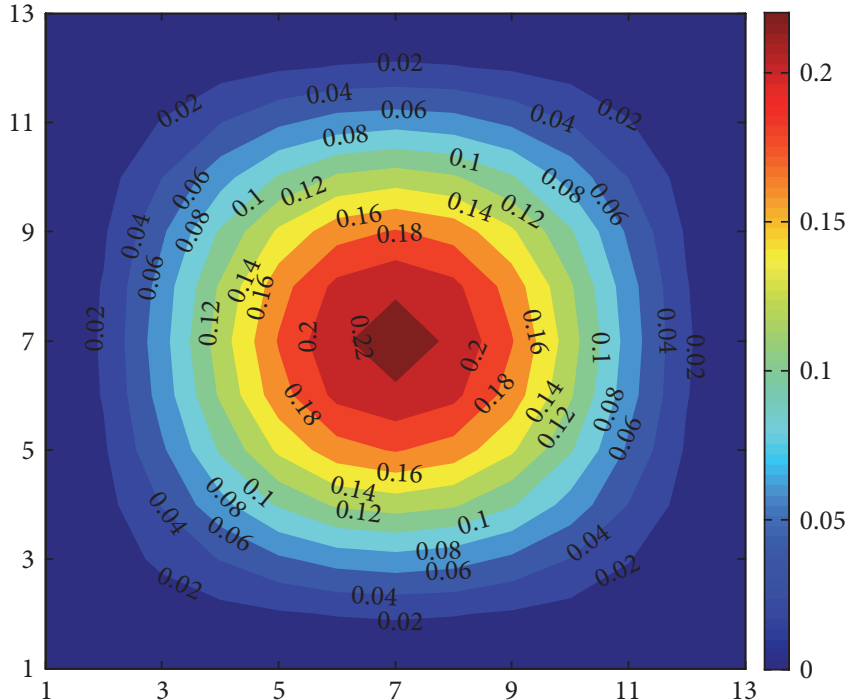

(b) The force is applied at point 1

FIGURE 21: The vibration normal velocity in the full coverage plate with CLD materials for second BC.

\section{Conclusion}

Finite element method and acoustic boundary element method are combined to predict sound radiation power of a closed acoustic cavity with a flexible surface treated with constrained layer damping (CLD). A topology optimization model is established with the objective of minimizing sound radiation power at the specified frequency. The evolutionary structural optimization (ESO) method and genetic algorithm (GA) with integer coding are employed to search optimal layouts of CLD materials bonded on the flexible plate in the cavity. The sound power sensitivity is formulated to determine the search direction in ESO optimization process.

A new boundary condition is defined for CLD/plate in the cavity besides a common boundary condition. Optimal layouts of CLD material with different rejections ratios are obtained and analyzed for the first mode using ESO and GA. It shows the two methods are feasible to solve the optimization problem. The computational time, the optimal layouts, and the minimum sound radiation power obtained using ESO and GA are compared. The results demonstrate that the ESO is more efficient to obtain deterministic and more practical optimal CLD material layouts for minimizing sound radiation power.

The optimal results obtained using ESO are discussed in detail. There is an optimal rejection ratio of CLD materials which makes the sound power reduction highest. It varies with variation of thickness of constrained layer material and viscoelastic material. The variation trend is opposite for the two boundary conditions. The locations of exciting force have also effect on the optimal layouts of CLD materials. The best rejection ratios of CLD materials lie in a small range but are different for the two boundary conditions.

\section{Conflicts of Interest}

The authors declare that there are no conflicts of interest regarding the publication of this paper.

\section{Acknowledgments}

This work was supported by the National Natural Science Foundation of China (no. 51275126).

\section{References}

[1] E. M. Kerwin, "Damping of flexural waves by a constrained viscoelastic layer," The Journal of the Acoustical Society of America, vol. 31, no. 7, pp. 952-962, 1959.

[2] H. Zheng, C. Cai, and X. M. Tan, "Optimization of partial constrained layer damping treatment for vibrational energy minimization of vibrating beams," Computers \& Structures, vol. 82, no. 5, pp. 2493-2507, 2004.

[3] H. Zheng, C. Cai, G. S. H. Pau, and G. R. Liu, "Minimizing vibration response of cylindrical shells through layout optimization of passive constrained layer damping treatments," Journal of Sound and Vibration, vol. 279, no. 3-5, pp. 739-756, 2005.

[4] Y.-C. Chen and S.-C. Huang, "An optimal placement of CLD treatment for vibration suppression of plates," International Journal of Mechanical Sciences, vol. 44, no. 8, pp. 1801-1821, 2002. 
[5] M. Alvelid, "Optimal position and shape of applied damping material," Journal of Sound and Vibration, vol. 310, no. 4-5, pp. 947-965, 2008.

[6] D. D. Zhang and L. Zheng, "Vibration characteristics analysis of CLD/plate based on the multi-objective optimization," Journal of Vibroengineering, vol. 17, no. 1, pp. 309-329, 2015.

[7] M. Ansari, A. Khajepour, and E. Esmailzadeh, "Application of level set method to optimal vibration control of plate structures," Journal of Sound and Vibration, vol. 332, no. 4, pp. 687-700, 2013.

[8] Y. N. Li, R. L. Xie, Y. Wang, and L. Zheng, “Topology optimization for constrained layer damping material in structures using ESO method," Journal of Chongqing University, vol. 8, pp. 1-6, 2010.

[9] Z. Fang and L. Zheng, “Topology optimization for minimizing frequency response of constrained layer damping plates," Journal of Vibroengineering, vol. 17, no. 6, pp. 2763-2780, 2015.

[10] H. Zheng and C. Cai, "Minimization of sound radiation from baffled beams through optimization of partial constrained layer damping treatment," Applied Acoustics, vol. 65, no. 5, pp. 501520, 2004.

[11] W. Zheng, T. Yang, Q. Huang, and Z. He, “Topology optimization of PCLD on plates for minimizing sound radiation at low frequency resonance," Structural and Multidisciplinary Optimization, vol. 53, no. 6, pp. 1231-1242, 2016.

[12] J. Du and N. Olhoff, "Minimization of sound radiation from vibrating bi-material structures using topology optimization," Structural and Multidisciplinary Optimization, vol. 33, no. 4-5, pp. 305-321, 2007.

[13] W. Akl, A. El-Sabbagh, K. Al-Mitani, and A. Baz, “Topology optimization of a plate coupled with acoustic cavity," International Journal of Solids and Structures, vol. 46, no. 10, pp. 2060 2074, 2009.

[14] L. Zheng, D. Zhang, C. Liu et al., "Topology optimization of a constrained layer damping plate coupled with an acoustical cavity," International Journal of Acoustics and Vibration, vol. 21, no. 4, pp. 394-405, 2016.

[15] R. D. Ciskowski and C. A. Brebbia, Boundary element methods in acoustics. Computational Mechanics Publications, Computational Mechanics Publications, Southampton Boston, Mass, USA, 1991.

[16] Y. M. Xie and G. P. Steven, "A simple evolutionary procedure for structural optimization," Computer \& Structures, vol. 49, no. 5, pp. 885-896, 1993.

[17] L. Zheng and Q. F. Zhu, "Topology Optimization for acoustic radiation of a constraint damping plate," Journal of Vibration and Shock, vol. 33, no. 5, pp. 91-96, 2014. 


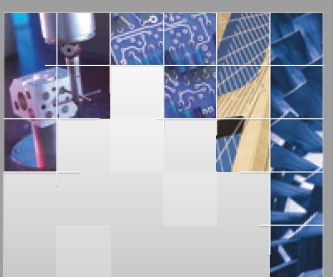

\section{Enfincering}
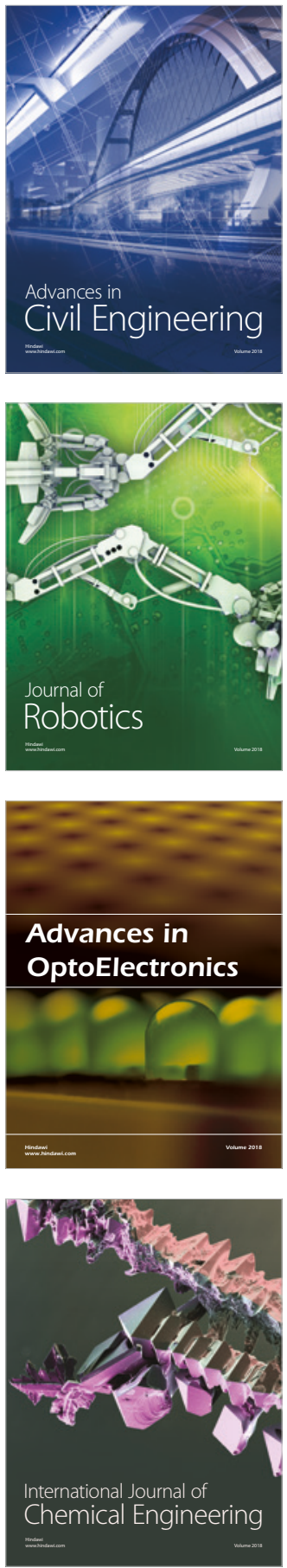

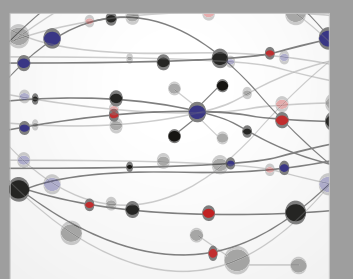

\section{Rotating \\ Machinery}

The Scientific World Journal

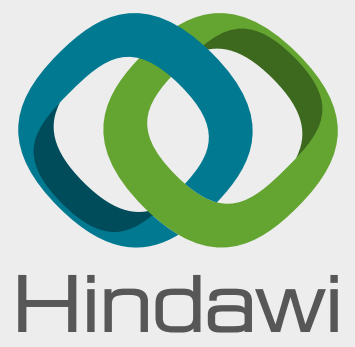

Submit your manuscripts at

www.hindawi.com
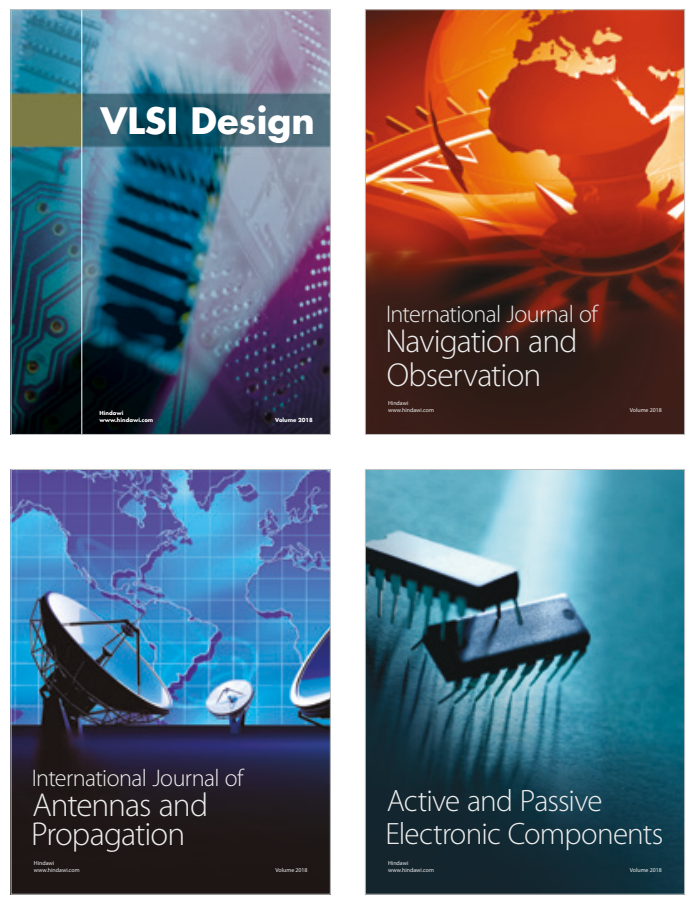
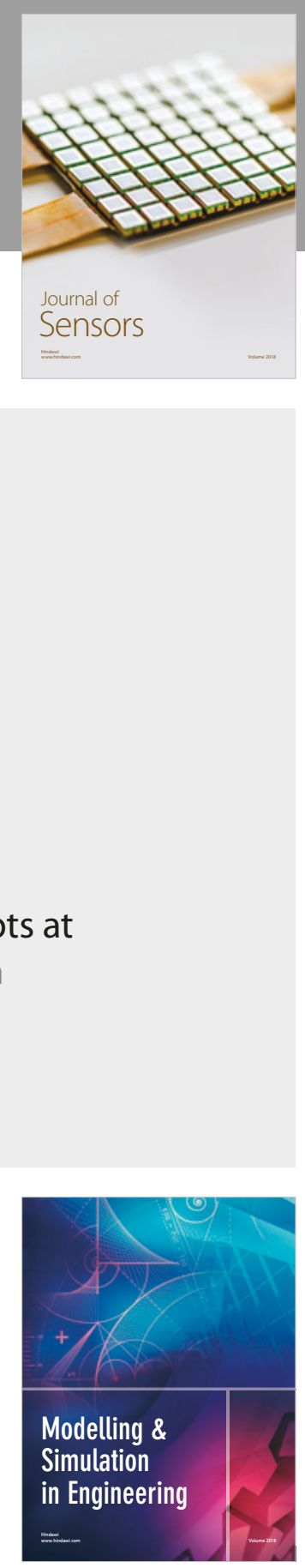

\section{Advances \\ Multimedia}
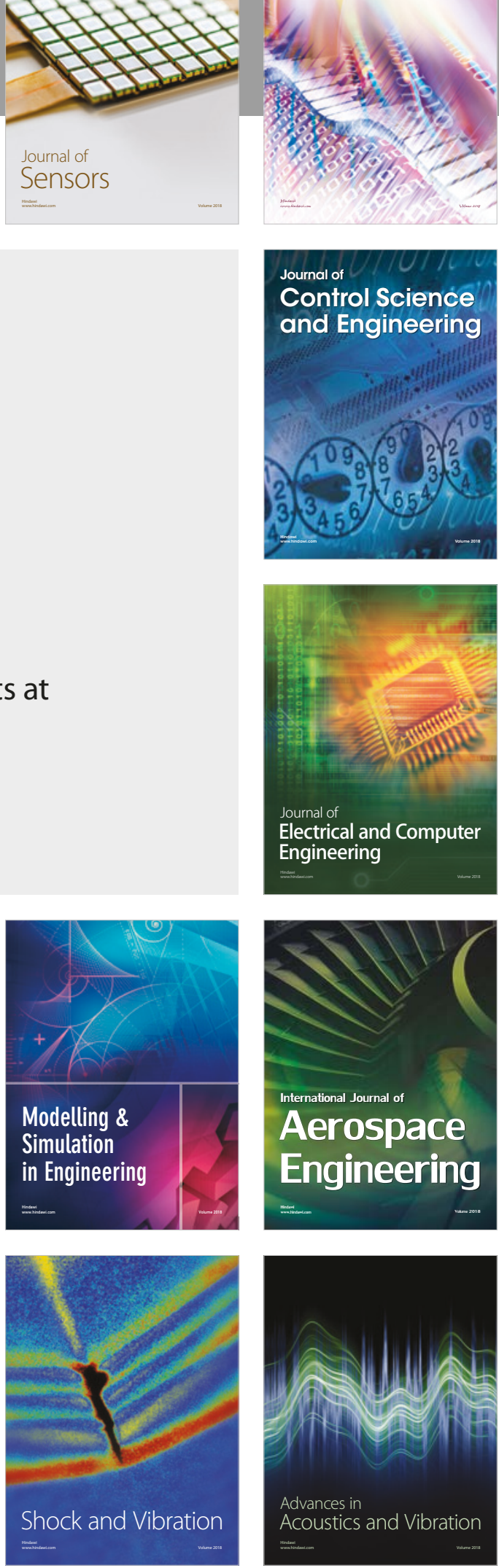